\title{
On the Condition Number Distribution of Complex Wishart Matrices
}

\author{
Michail Matthaiou, Member, IEEE, Matthew R. McKay, Member, IEEE, \\ Peter J. Smith, Senior Member, IEEE, and Josef A. Nossek, Fellow, IEEE
}

\begin{abstract}
This paper investigates the distribution of the condition number of complex Wishart matrices. Two closely related measures are considered: the standard condition number (SCN) and the Demmel condition number (DCN), both of which have important applications in the context of multiple-input multipleoutput (MIMO) communication systems, as well as in various branches of mathematics. We first present a novel generic framework for the SCN distribution which accounts for both central and non-central Wishart matrices of arbitrary dimension. This result is a simple unified expression which involves only a single scalar integral, and therefore allows for fast and efficient computation. For the case of dual Wishart matrices, we derive new exact polynomial expressions for both the SCN and DCN distributions. We also formulate a new closed-form expression for the tail SCN distribution which applies for correlated central Wishart matrices of arbitrary dimension and demonstrates an interesting connection to the maximum eigenvalue moments of Wishart matrices of smaller dimension.

Based on our analytical results, we gain valuable insights into the statistical behavior of the channel conditioning for various MIMO fading scenarios, such as uncorrelated/semi-correlated Rayleigh fading and Ricean fading.
\end{abstract}

Index Terms-MIMO systems, complex Wishart matrices, condition number, joint eigenvalue distribution.

\section{INTRODUCTION}

$\mathbf{O}$ VER the past decade, multiple-input multiple-output (MIMO) systems have been at the forefront of wireless communications research and development, due to their huge potential for delivering significant capacity gains compared with conventional systems. The capacity and performance of practical MIMO transmission schemes are often dictated by the statistical eigenproperties of the instantaneous channel correlation matrix ${ }^{1} \mathbf{W}=\mathbf{H H}^{\dagger}$ or $\mathbf{W}=\mathbf{H}^{\dagger} \mathbf{H}$, with $\mathbf{H}$ denoting the matrix of random MIMO channel gains. The

Paper approved by N. Al-Dhahir, the Editor for Space-Time, OFDM and Equalization of the IEEE Communications Society. Manuscript received June 10, 2009; revised October 21, 2009.

M. Matthaiou and J. A. Nossek are with the Institute for Circuit Theory and Signal Processing, Technische Universität München (TUM), Arcistrasse 21, 80333, Munich, Germany (e-mail: \{matthaiou, nossek\}@nws.ei.tum.de).

M. R. McKay is with the Department of Electronic and Computer Engineering, Hong Kong University of Science and Technology (HKUST), Clear Water Bay, Kowloon, Hong Kong (e-mail: eemckay@ust.hk).

P. J. Smith is with the Department of Electrical and Computer Engineering, University of Canterbury, Private Bag 4800, Christchurch, New Zealand (email: peter.smith@canterbury.ac.nz).

Part of this paper has been presented at the IEEE International Conference on Communications (ICC), Cape Town, South Africa, May 2010.

The work of M. R. McKay was supported by the research grant DAG S08/09.EG04.

Digital Object Identifier 10.1109/TCOMM.2010.06.090328

${ }^{1}$ The notation $(\cdot)^{\dagger}$ denotes the conjugate-transpose operation. matrix $\mathbf{H}$ is typically modeled via a complex Gaussian distribution with mean and covariance structure defined by the system configuration (eg. antenna spacing) and the nature of the surrounding environment (eg. line-of-sight $(\mathrm{LoS})$ ). As such, $\mathbf{W}$ is known to follow a complex Wishart distribution.

In recent years, the statistical properties of Wishart matrices have been extensively studied and applied to a large number of MIMO applications. For example, the unordered eigenvalue distributions and determinant properties of Wishart matrices have been derived in [1-16] and applied to explore the ergodic capacity of the MIMO channel under different conditions, whereas ordered/marginal eigenvalue distributions have been derived in [17-26] and used to investigate the performance of MIMO beamforming strategies. Many other statistical properties of Wishart matrices, such as those involving Gaussian quadratic forms, have also been applied to MIMO analysis in different contexts (eg. [27]). For a contemporary review of random matrix theory and its application to wireless communications, see [28].

In this paper, we investigate the distribution of the condition number of Wishart matrices, considering two closely related measures: (i) the standard condition number ( $\mathrm{SCN})$, defined as the ratio of the largest to the smallest eigenvalue, and (ii) the Demmel condition number (DCN), which returns the ratio of the matrix trace to the minimum eigenvalue [29]. Both of these condition numbers give a measure of the relative conditioning (or rank-deficiency) of a matrix, and the statistical properties of both have been shown to be important for a variety of applications. For example, the SCN has been used to indicate the degree of multipath activity in MIMO communication channels [30,31], and has been shown to have a close connection with the performance of linear MIMO receivers in spatial multiplexing systems [3234]; further, it has been adopted to formulate novel spectrum sensing algorithms in cognitive radio applications [35-37]. The distribution of the SCN also has many applications beyond the realm of wireless communications; for example, it has been employed in classical linear algebra for investigating the sensitivity of matrix inversion problems to perturbation errors and the convergence rate of iterative schemes [38]. The distribution of the DCN, on the other hand, has been employed in the context of MIMO to determine diversity-multiplexing switching criteria for adaptive MIMO systems [39], and also in the design of link adaptation protocols [40].

Despite the importance of the SCN and DCN of Wishart matrices, little is still known about their statistical properties. In particular, this issue was first considered in the pioneering 
work of Edelman [41] whose SCN analysis focused on $2 \times 2$ (i.e. dual) uncorrelated central Wishart matrices with arbitrary degrees of freedom (DoF), and also on asymptotic distributions, valid for infinitely large dimensions. Some results on the DCN were also presented, which gave asymptotic approximations for the DCN distribution under certain conditions. In [42-44], the authors proposed simple efficient bounds on the high-tails of the SCN distributions, which again focused primarily on uncorrelated central Wishart matrices. The SCN probability density function (PDF) for the uncorrelated central case was recently presented in tensor form in [45], using the results of [46] for the ordered eigenvalues of Wishart matrices. The SCN analysis of [41] was extended in [47] to allow for matrices of arbitrary size and arbitrary correlation structures; however, the final result for the SCN PDF was given as a complicated expression involving an infinite series of zonal polynomials, and is thus difficult to manage. Finally, the exact SCN distributions for dual complex non-central Wishart matrices with two DoF were recently presented in [48].

In this paper, we first present a novel generic framework for the exact SCN distribution for different classes of complex Wishart matrices. This key result is a unified expression which applies for both central and non-central Wishart matrices of arbitrary dimension (for the central case, allowing for arbitrary correlation structure), and involves only a single scalar integral which allows for fast and efficient computation. We note that the analysis of non-central Wishart matrices is based on both an exact infinite series representation as well as on a tractable approximation which extensively simplifies the mathematical formulations. For the specific case of dual uncorrelated and correlated central Wishart matrices, we also derive exact polynomial expressions for the SCN and DCN distributions. Moreover, for correlated central Wishart matrices of arbitrary dimension, we propose a new closed-form expression for the SCN tail distribution. This result generalizes recent work from [44] to account for arbitrary correlation structures, and reveals an interesting connection between the tail behavior of the SCN distribution and the maximum eigenvalue moments of a Wishart matrix of smaller dimension. Based on our analytical results, we gain valuable insights into the statistical behavior of the channel conditioning for various MIMO fading scenarios, such as uncorrelated/semi-correlated Rayleigh and Ricean fading.

The rest of the paper is organized as follows. In Section II, three common MIMO Gaussian fading models are introduced, along with their connections to different classes of complex Wishart distributions. The corresponding joint ordered eigenvalue distributions are also addressed. In Section III, our new results on the SCN distribution are presented, whereas Section IV deals with the DCN. In Section V, the theoretical results are validated via Monte-Carlo simulations, while Section VI concludes the paper and summarizes the key findings.

Notation: We use upper and lower case boldface for matrices and vectors, respectively. The $n \times n$ identity matrix is expressed as $\mathbf{I}_{n}$, while the $(i, j)$-th entry of a matrix $\mathbf{A}$ is denoted as $a_{i, j}$. The symbol $(\cdot)^{T}$ denotes the transpose, $\operatorname{tr}(\cdot)$ yields the matrix $\operatorname{trace}$, etr $(\cdot)$ is shorthand for $\exp (\operatorname{tr}(\cdot))$, and $|\cdot|$ represents the determinant. The symbol $\stackrel{d}{\sim}$ denotes "distributed as", while $\sim$ will be used for asymptotic notation.

\section{Mimo Channels Models And their Eigenvalue DISTRIBUTIONS}

Consider a MIMO system equipped with $N_{t}$ transmit and $N_{r}$ receive antennas. The wireless channel can be effectively characterized by the matrix $\mathbf{H} \in \mathbb{C}^{N_{r} \times N_{t}}$, whose entries represent the complex responses between each antenna pair. For most performance measures of interest, such as the ergodic and outage capacities $[1,2,4,5,20,25]$, the diversity-multiplexing trade-off [49], and symbol error rate of practical transmission schemes $[18,19,21,26,27]$, the effect of the channel is reflected via the statistical properties of the instantaneous MIMO correlation matrix

$$
\mathbf{W} \triangleq\left\{\begin{array}{lll}
\mathbf{H H}^{\dagger}, & \text { if } \quad N_{r} \leq N_{t} \\
\mathbf{H}^{\dagger} \mathbf{H}, & \text { if } \quad N_{r}>N_{t} .
\end{array}\right.
$$

The channel matrix $\mathbf{H}$ is typically modeled as complex Gaussian. In this paper, we consider three common classes of channels, for which $\mathbf{W} \in \mathbb{C}^{s \times s}$ follows a Wishart distribution with $t$ DoF, where $s \triangleq \min \left(N_{t}, N_{r}\right)$ and $t \triangleq \max \left(N_{t}, N_{r}\right)$. Note that, in all cases, the channel is normalized such that $E[\operatorname{tr}(\mathbf{W})]=N_{r} N_{t}$. Hereafter, we wiil use $\mathbf{H}_{w}$ to represent an $N_{r} \times N_{t}$ matrix whose entries are i.i.d. $\stackrel{\text { d }}{\sim} \mathcal{C N}(0,1)$ random variables.

Definition 1 (Uncorrelated Rayleigh Fading): The uncorrelated Rayleigh model is valid when the antenna spacings and/or the angular spreads are high enough to induce independent fading, and there is no LoS path between the transmitter and receiver. Under these conditions,

$$
\mathbf{H}=\mathbf{H}_{w}
$$

and $\mathbf{W}$ is uncorrelated central Wishart with $t$ DoF, commonly denoted as $\mathbf{W} \stackrel{\mathrm{d}}{\sim} \mathcal{C W}_{s}\left(t, \mathbf{I}_{s}\right)$ [50].

Definition 2 (Semi-Correlated Rayleigh Fading): In practice, the MIMO spatial subchannels are often correlated due to the limited angular spreads or restrictions on the array sizes. The effect of spatial correlation can be reflected as follows

$$
\mathbf{H}= \begin{cases}\boldsymbol{\Sigma}_{s}^{1 / 2} \mathbf{H}_{w}, & \text { if } \quad N_{r} \leq N_{t} \\ \mathbf{H}_{w} \boldsymbol{\Sigma}_{s}^{1 / 2}, & \text { if } \quad N_{r}>N_{t}\end{cases}
$$

where $\boldsymbol{\Sigma}_{s} \in \mathbb{C}^{s \times s}>\mathbf{0}$ denotes the spatial correlation matrix. Note that here we have assumed that correlation occurs only at the side with minimum number of antennas ${ }^{2}$, in which case $\mathbf{W}$ is semi-correlated central Wishart, expressed as $\mathbf{W} \stackrel{\mathrm{d}}{\sim} \mathcal{C W}_{s}\left(t, \boldsymbol{\Sigma}_{s}\right)$

Definition 3 (Uncorrelated Ricean Fading): This scenario is relevant when there is a direct LoS path between the transmitter and receiver. Then, the channel matrix consists of a deterministic component, $\mathbf{H}_{\mathrm{L}}$, and a Rayleigh-distributed random component, $\mathbf{H}_{w}$, which accounts for the scattered signals, or

$$
\mathbf{H}=\sqrt{\frac{K_{r}}{K_{r}+1}} \mathbf{H}_{\mathrm{L}}+\sqrt{\frac{1}{K_{r}+1}} \mathbf{H}_{w}
$$

\footnotetext{
${ }^{2}$ Correlation at the side with the maximum number of antennas can also be handled, however the notation is more cumbersome. Thus, we choose to omit the explicit presentation of this scenario throughout the paper.
} 
where $K_{r}$ stands for the Ricean $K$-factor, representing the ratio of the power of the deterministic component to the power of the fading component. Clearly, $\mathbf{H}$ has a non-zero mean, given by $E\{\mathbf{H}\}=\mathbf{M}=\sqrt{K_{r} /\left(K_{r}+1\right)} \mathbf{H}_{\mathrm{L}}$. In this case, $\mathbf{W}$ is uncorrelated non-central Wishart, usually expressed as $\mathbf{W} \stackrel{\mathrm{d}}{\sim} \mathcal{C W}_{s}\left(t, \varepsilon^{2} \mathbf{I}_{s}, \boldsymbol{\Omega}\right)$, where $\varepsilon=1 / \sqrt{K_{r}+1}$ is a power scaling factor, and

$$
\boldsymbol{\Omega}= \begin{cases}\varepsilon^{-2} \mathbf{M M}^{\dagger}, & \text { if } N_{r} \leq N_{t} \\ \varepsilon^{-2} \mathbf{M}^{\dagger} \mathbf{M}, & \text { if } N_{r}>N_{t}\end{cases}
$$

is the non-centrality matrix. For the sake of consistency with previous studies, we consider a scaled version of $\mathbf{W}$, that is $\mathbf{S}=\varepsilon^{-2} \mathbf{W} \stackrel{\mathrm{d}}{\sim} \mathcal{C W}_{s}\left(t, \mathbf{I}_{s}, \boldsymbol{\Omega}\right)[18,19,50]$. Note that $\mathbf{S}$ and $\mathbf{W}$ have, by definition, the same SCN.

Explicit solutions can sometimes be obtained for the distributional properties of non-central Wishart matrices. However, they are usually more complicated than the corresponding expressions for central Wishart matrices. In this light, the following well-known result, which approximates a non-central Wishart distribution with a central Wishart distribution of modified correlation structure, will be particularly useful.

Lemma 1 ([51]): A complex semi-correlated central Wishart matrix $\mathbf{W} \stackrel{\mathrm{d}}{\sim} \mathcal{C W}_{s}\left(t, \widehat{\boldsymbol{\Sigma}}_{s}\right)$, with the effective correlation matrix being $\widehat{\boldsymbol{\Sigma}}_{s}=\varepsilon^{2} \mathbf{I}_{s}+(1 / t) \boldsymbol{\Omega}$, has the same first-order moments and second-order moments differing by $(1 / t) \Omega$, as the complex non-central Wishart matrix $\mathbf{W} \stackrel{\mathrm{d}}{\sim} \mathcal{C} \mathcal{W}_{s}\left(t, \varepsilon^{2} \mathbf{I}_{s}, \mathbf{\Omega}\right)$

\section{A. Joint Ordered Eigenvalue Distributions}

The instantaneous MIMO correlation matrix $\mathbf{W}$ (or $\mathbf{S}$ ) is Hermitian and positive definite, hence its eigenvalues are real and strictly positive. Let us denote these eigenvalues by the vector $\boldsymbol{\lambda} \triangleq\left[\lambda_{1}, \lambda_{2}, \ldots, \lambda_{s}\right]^{T}$, with $\lambda_{1} \geq \lambda_{2} \ldots \geq \lambda_{s}>0$. For each of the three channel models introduced in Definitions 13 , the corresponding joint ordered eigenvalue distributions admit the following generic form [8,22, 23, 52]

$$
f(\boldsymbol{\lambda})=K|\boldsymbol{\Phi}(\boldsymbol{\lambda})| \times|\boldsymbol{\Psi}(\boldsymbol{\lambda})| \prod_{\ell=1}^{s} \xi\left(\lambda_{\ell}\right) .
$$

Table I on the next page lists the associated parameters for each of the channel scenarios considered. Note that here we adopt similar notation as in [23]. In Table $\mathrm{I}, \mathbf{V}_{1}(\boldsymbol{\lambda})$ is a Vandermonde matrix with $(i, j)$-th entry $\lambda_{j}^{i-1}$; the vectors $\boldsymbol{\sigma} \triangleq\left[\sigma_{1}, \sigma_{2}, \ldots, \sigma_{s}\right]^{T}$ and $\boldsymbol{\omega} \triangleq\left[\omega_{1}, \omega_{2}, \ldots, \omega_{s}\right]^{T}$ contain the non-zero ordered eigenvalues (assumed distinct) of $\boldsymbol{\Sigma}_{s}$ and $\boldsymbol{\Omega}$, respectively, i.e. $\sigma_{1}>\sigma_{2} \ldots>\sigma_{s}>0$ and $\omega_{1}>\omega_{2} \ldots>$ $\omega_{s}>0$; also, $\mathbf{F}(\boldsymbol{\lambda}, \boldsymbol{\omega})$ and $\mathbf{E}(\boldsymbol{\lambda}, \boldsymbol{\sigma})$ have $(i, j)$-th entries ${ }_{0} \mathcal{F}_{1}\left(t-s+1 ; \lambda_{j} \omega_{i}\right)$ and $\exp \left(-\lambda_{j} / \sigma_{i}\right)$ respectively, with ${ }_{0} \mathcal{F}_{1}(\cdot ; \cdot)$ denoting the standard generalized hypergeometric function [53, Eq. (9.14.1)]. Note that in all cases, the $(i, j)$-th element of $\boldsymbol{\Phi}(\boldsymbol{\lambda})$ and $\boldsymbol{\Psi}(\boldsymbol{\lambda})$ has the same general form:

$$
\{\boldsymbol{\Phi}(\boldsymbol{\lambda})\}_{i, j}=\phi_{i}\left(\lambda_{j}\right), \quad\{\boldsymbol{\Psi}(\boldsymbol{\lambda})\}_{i, j}=\psi_{i}\left(\lambda_{j}\right)
$$

with $\phi_{i}(\cdot)$ and $\psi_{i}(\cdot)$ defined according to the specific channel scenario, as described above. The generic eigenvalue distribution (6) will be particularly important for deriving the SCN distribution in the following.

\section{Statistics of the Standard Condition Number}

The SCN, defined as

$$
z \triangleq \frac{\lambda_{1}}{\lambda_{s}}, \quad z \geq 1
$$

is a metric which determines the invertibility of a matrix. A condition number close to one indicates a well-conditioned full-rank matrix with almost equal eigenvalues, whereas a very high condition number implies a near rank-deficient matrix. As was previously stated, the SCN arises in various areas in MIMO communications, as well as other mathematical fields such as numerical analysis and linear algebra. In general, the statistical properties of the SCN of MIMO communication channels have not been well investigated. In this section, we present new expressions for the distribution of the SCN, which embraces each of the channel models introduced in Section II.

\section{A. General Framework for the SCN Distribution of Wishart Matrices}

The following theorem establishes a new general framework for the cumulative distribution function (CDF) of the SCN of different classes of Wishart matrices, which applies for arbitrary matrix dimensions and arbitrary DoF. This constitutes a key contribution of the paper.

Theorem 1: The CDF, $F_{z}(x)=\operatorname{Pr}(z \leq x)$, with $x \geq$ 1 , of the SCN of an uncorrelated central Wishart matrix, $\mathbf{W} \stackrel{\mathrm{d}}{\sim} \mathcal{C} \mathcal{W}_{s}\left(t, \mathbf{I}_{s}\right)$, semi-correlated central Wishart matrix, $\mathbf{W} \stackrel{\mathrm{d}}{\sim} \mathcal{C W}_{s}\left(t, \boldsymbol{\Sigma}_{s}\right)$, and uncorrelated non-central Wishart matrix, $\mathbf{S} \stackrel{\mathrm{d}}{\sim} \mathcal{C} \mathcal{W}_{s}\left(t, \mathbf{I}_{s}, \boldsymbol{\Omega}\right)$, can be represented as ${ }^{3}$

$F_{z}(x)=K \sum_{\ell=1}^{s} \int_{0}^{\infty}\left|\left[\begin{array}{ll}\int_{\lambda_{s}}^{x \lambda_{s}} \phi_{i}(u) \psi_{j}(u) \xi(u) d u, & i \neq \ell \\ \phi_{i}\left(\lambda_{s}\right) \psi_{j}\left(\lambda_{s}\right) \xi\left(\lambda_{s}\right), & i=\ell\end{array}\right]\right| d \lambda_{s}$

with $\phi_{i}(\cdot)$ and $\psi_{i}(\cdot)$ defined as in (7), while $K$ is the normalization constant given in Table I.

Proof: See Appendix A.

It is important to note that all integrals inside the determinant admit a closed-form solution, for all cases of interest. Thus, only a single integration is required, whose numerical evaluation is more robust and efficient compared to conventional Monte-Carlo simulations. The closed-form solutions for the definite integrals in (9) are summarized in Table II, where we have made use of [53, Eq. (3.381.1)] and [18, Eq. (4)]. In these expressions, $\gamma(a, x)$ denotes the lower incomplete gamma function [53, Eq. (8.350.1)], and $Q_{p, q}(a, b)$ is the Nuttall $Q$-function defined as [54]

$$
Q_{p, q}(a, b)=\int_{b}^{\infty} x^{p} \exp \left(-\frac{x^{2}+a^{2}}{2}\right) I_{q}(a x) d x
$$

with $I_{q}(\cdot)$ denoting the $q$-th order modified Bessel function of the first kind.

We now take a deeper look at the numerical evaluation of the non-central case. We first recall that since the sum of the indices of the Nuttall- $Q$ function in Table II is always odd, a closed-form representation is given in [55, Eq. (13)]

\footnotetext{
${ }^{3}$ Here we adopt the compact notation for the determinant of a matrix, written in terms of the $(i, j)$-th element.
} 
TABLE I

JoINT EIGENVALUE PDF OF COMPLEX WiShaRT MATRICES: PARAMETERS AND NORMALIZATION CONSTANTS

\begin{tabular}{c||c|c|c|c} 
& $\mathbf{\Phi}(\boldsymbol{\lambda})$ & $\mathbf{\Psi}(\boldsymbol{\lambda})$ & $\xi\left(\lambda_{\ell}\right)$ & $K$ \\
\hline \hline Uncorrelated central (UC) & $\mathbf{V}_{1}(\boldsymbol{\lambda})$ & $\mathbf{V}_{1}(\boldsymbol{\lambda})$ & $\lambda_{\ell}^{t-s} e^{-\lambda_{\ell}}$ & $K_{u c}=\left[\prod_{i=1}^{s}(s-i) ! \prod_{j=1}^{s}(t-j) !\right]^{-1}$ \\
Semi-correlated central (CC) & $\mathbf{V}_{1}(\boldsymbol{\lambda})$ & $\mathbf{E}(\boldsymbol{\lambda}, \boldsymbol{\sigma})$ & $\lambda_{\ell}^{t-s}$ & $K_{c c}=\prod_{i=1}^{s} \frac{1}{\sigma_{i}^{t}(t-i) !} \prod_{i<j}^{\sigma_{i} \sigma_{j}}$ \\
& & & & \\
Uncorrelated non-central (UN) & $\mathbf{V}_{1}(\boldsymbol{\lambda})$ & $\mathbf{F}(\boldsymbol{\lambda}, \boldsymbol{\omega})$ & $\lambda_{\ell}^{t-s} e^{-\lambda_{\ell}}$ & $K_{u n}=(t-s) !^{-s} \operatorname{etr}(-\boldsymbol{\omega})\left|\mathbf{V}_{1}(\boldsymbol{\omega})\right|^{-1}$ \\
\hline \hline
\end{tabular}

in terms of Marcum $Q$-functions and a finite weighted sum of Bessel functions. Hence, a direct evaluation of (9) involves either Nuttall- $Q$ or Marcum- $Q$ functions both of which are somehow difficult to manipulate. In order to simplify the procedure, a second alternative is to apply the central/noncentral approximation of Lemma 1 on (4), so that the resulting SCN CDF expression does not involve the Nuttall- $Q$ function. While this approximation simplifies the non-central analysis, its accuracy deteriorates at high Ricean $K$-factors.

The third alternative, which still leads to an exact solution, is to expand the involved hypergeometric function in the integrand of (9), according to [53, Eq. (9.14.1)]

$$
{ }_{0} \mathcal{F}_{1}(m ; x)=\sum_{k=0}^{\infty} \frac{z^{k}(m-1) !}{k !(m+k-1) !}
$$

so that the corresponding definite integral inside the determinant for the non-central case becomes

$$
\begin{aligned}
\mathcal{I}_{i, j}=\sum_{k=0}^{\infty}\left(\frac{(t-s) ! \omega_{j}^{k}}{k !(t-s+k) !}\right) & \left(\gamma\left(t-s+k+i, x \lambda_{s}\right)\right. \\
& \left.-\gamma\left(t-s+k+i, \lambda_{s}\right)\right) .
\end{aligned}
$$

This expression converges quickly for most practical MIMO configurations and $K$-factors, e.g. $t \leq 20$ and $K_{r} \leq 10 \mathrm{~dB}$, and thus can be truncated to a finite number of terms while still yielding good accuracy. This accuracy will be demonstrated through simulations in Section V.

\section{B. Exact Expressions for the SCN Distribution of Dual Wishart Matrices}

We now focus on the case of dual Wishart matrices with arbitrary DoF, i.e. $s=2$ and $t \geq 2$. This scenario is particularly important for MIMO systems, where size/implementation cost limitations of the mobile terminals typically restrict the numbers of antennas to be small (e.g. hand-held devices). In the following, we present a closed-form polynomial expressions for the CDF and PDF of the SCN for the case of dual uncorrelated central Wishart matrices. We then consider the more general case of semi-correlated central Wishart.

Theorem 2: The $\mathrm{CDF}$ of the $\mathrm{SCN}$ of $\mathbf{W} \stackrel{\mathrm{d}}{\sim} \mathcal{C W}_{2}\left(t, \mathbf{I}_{2}\right)$ is given by

$$
F_{z}(x)=K_{u c}\left(p_{1}(x)-p_{1}(1)\right), \quad x \geq 1
$$

TABLE II

CLOSED-FORM EXPRESSIONS FOR THE DEFINITE INTEGRAL IN (9)

\begin{tabular}{c|c} 
& $\mathcal{I}_{i, j}=\int_{\lambda_{s}}^{x \lambda_{s}} \phi_{i}(u) \psi_{j}(u) \xi(u) d u$ \\
\hline \hline UC & $\gamma\left(t-s+i+j-1, x \lambda_{s}\right)-\gamma\left(t-s+i+j-1, \lambda_{s}\right)$ \\
\hline CC & $\left.\left(\gamma\left(t-s+i, x \lambda_{s} / \sigma_{j}\right)-\gamma(t-s+i), \lambda_{s} / \sigma_{j}\right)\right) \sigma_{j}^{t-s+i}$ \\
\hline & $(t-s) ! e^{\omega_{j}} \omega_{j}^{(s-t) / 2} 2^{(s-t-2 i+2) / 2}$ \\
UN & $\times\left(Q_{t-s+2 i-1, t-s}\left(\sqrt{2 \omega_{j}}, \sqrt{2 \lambda_{s}}\right)\right.$ \\
& $\left.-Q_{t-s+2 i-1, t-s}\left(\sqrt{2 \omega_{j}}, \sqrt{2 x \lambda_{s}}\right)\right)$ \\
\hline \hline
\end{tabular}

where

$p_{1}(y)=\Delta_{1}(t, t-1, y)-2 \Delta_{1}(t-1, t, y)+\Delta_{1}(t-2, t+1, y)$

and

$$
\Delta_{1}(m, n, y)=(n-1) !\left(m !-\sum_{k=0}^{n-1} \frac{(m+k) ! y^{k}}{k !(y+1)^{m+k+1}}\right) .
$$

Proof: We start by particularizing (9) to the uncorrelated central Wishart scenario using Table I. Then, by substituting $s=2$ and expanding the determinant gives

$$
\begin{gathered}
F_{z}(x)=K_{u c}\left\{\int _ { 0 } ^ { \infty } e ^ { - \lambda _ { 2 } } \lambda _ { 2 } ^ { t - 2 } \left(\lambda_{2}^{2} \int_{\lambda_{2}}^{x \lambda_{2}} u^{t-2} e^{-u} d u\right.\right. \\
\left.\left.-2 \lambda_{2} \int_{\lambda_{2}}^{x \lambda_{2}} u^{t-1} e^{-u} d u+\int_{\lambda_{2}}^{x \lambda_{2}} u^{t} e^{-u} d u\right) d \lambda_{2}\right\} .
\end{gathered}
$$

The result follows by integrating using [53, Eq. (3.351.1)] and [53, Eq. (3.351.3)], and simplifying.

Corollary 1: The PDF of the SCN of $\mathbf{W} \stackrel{\mathrm{d}}{\sim} \mathcal{C W}_{2}\left(t, \mathbf{I}_{2}\right)$ is given by

$$
\begin{aligned}
f(z)=K_{u c}( & \Delta_{2}(t, t-1, z)-2 \Delta_{2}(t-1, t, z) \\
& \left.+\Delta_{2}(t-2, t+1, z)\right), \quad z \geq 1
\end{aligned}
$$

where

$\Delta_{2}(m, n, y)=-(n-1) ! \sum_{k=0}^{n-1} \frac{(m+k) ! y^{k-1}(k-(m+1) y)}{k !(y+1)^{m+k+2}}$.

Proof: Obtained by differentiating (13).

Note that an alternative expression for the SCN PDF of uncorrelated central Wishart matrices was also originally given 
by Edelman in [41, Eq. (7.2)]. We have, however, included the result (15), derived using different methods and in the context of our new general framework, for the sake of completeness. In the following, we derive exact closed-form expressions for the CDF and PDF of the SCN for dual semi-correlated central Wishart matrices. To the best of our knowledge, these results are new.

Theorem 3: The $\mathrm{CDF}$ of the $\mathrm{SCN}$ of $\mathbf{W} \stackrel{\mathrm{d}}{\sim} \mathcal{C W}_{2}\left(t, \boldsymbol{\Sigma}_{2}\right)$ is given as

$$
F_{z}(x)=K_{c c}\left(p_{2}(x)-p_{2}(1)\right), \quad z \geq 1
$$

where $p_{2}(y)$ is defined in (17) while

$$
\begin{aligned}
& \Delta_{3}(m, n, \mu, \nu, y) \\
& =(n-1) !\left(\frac{m !}{\mu^{n} \nu^{m+1}}-\sum_{k=0}^{n-1} \frac{(m+k) ! y^{k}}{k ! \mu^{n-k}(\mu y+\nu)^{m+k+1}}\right) .
\end{aligned}
$$

Proof: We start by particularizing (9) to the semicorrelated central Wishart scenario using Table I. Then, substituting $s=2$ and expanding the determinant gives (18). Integrating using [53, Eq. (3.351.1)] and [53, Eq. (3.351.3)], we readily obtain (16).

Corollary 2: The PDF of the SCN of $\mathbf{W} \stackrel{\mathrm{d}}{\sim} \mathcal{C W}_{2}\left(t, \boldsymbol{\Sigma}_{2}\right)$ is given in (19) at the bottom of the page while

$$
\begin{aligned}
& \Delta_{4}(m, n, \mu, \nu, y) \\
& =-(n-1) ! \sum_{k=0}^{n-1} \frac{(m+k) ! y^{k-1}(k \nu-\mu(m+1) y)}{k ! \mu^{n-k}(\mu y+\nu)^{m+k+2}} .
\end{aligned}
$$

Proof: Obtained by differentiating (16).

In contrast to the dual central uncorrelated and semicorrelated Wishart scenarios considered above, the case of dual uncorrelated non-central Wishart matrices does not lend itself to a tractable representation. This is due, primarily, to the cross-products of hypergeometric and Nuttall- $Q$ functions that arise upon the determinant expansion in (9). However, an infinite series representation can be obtained as in [48],

${ }^{4}$ Reference [41] adopts a slightly different definition of the SCN, given by the square root of (8). while a closed-form approximation for the SCN CDF/PDF of dual complex non-central Wishart matrices is directly obtained from Theorem 3 and Corollary 2 respectively, upon application of Lemma 1 (i.e. by invoking the central/non-central Wishart approximation).

\section{High-Tail Distribution for the SCN of Wishart Matrices}

We now investigate the probability of experiencing an "extremely large" condition number. This is an important issue for various practical applications. For example, in the MIMO context, it predicts the probability of experiencing an extremely poor channel due to fading. Moreover, since the performance of MIMO linear receivers is intimately related to the channel condition number, the asymptotic analysis provides insights into the likelihood of such receivers failing in practice [32]. The asymptotic analysis is also important for characterizing the performance and complexity of adaptive MIMO detectors [34] or lattice-aided MIMO receivers [56], and has direct implications for the design of detection thresholds for spectrum sensing algorithms (see for instance [3537]).

The high-tail distribution of uncorrelated central Wishart matrices was recently established in [44, Theorem 3.2], which revealed an interesting relationship in terms of the maximum eigenvalue moments of a smaller Wishart matrix. Using a similar technique to that of [44], we herein generalize that result to the case of semi-correlated central Wishart matrices, and demonstrate a similar interesting relationship in terms of maximum eigenvalue moments.

We first require the following lemma:

Lemma 2: Let $f\left(\lambda_{1}, \lambda_{s}\right)$ be the joint density of the maximum and minimum eigenvalues of $\mathbf{W} \stackrel{\mathrm{d}}{\sim} \mathcal{C} \mathcal{W}_{s}\left(t, \boldsymbol{\Sigma}_{s}\right)$. Then, for fixed $\lambda_{1}$,

$$
\begin{aligned}
& f\left(\lambda_{1}, \lambda_{s}\right) \sim f_{\text {asy }}\left(\lambda_{1}, \lambda_{s}\right) \\
& =(t-s+1) \lambda_{s}^{t-s} \sum_{\ell=1}^{s} \mathcal{K}_{\ell} g_{s-1, t}\left(\lambda_{1}, \sigma_{[\ell]}\right), \quad\left(\lambda_{s} \rightarrow 0\right)
\end{aligned}
$$

$$
\begin{aligned}
p_{2}(y) & =\Delta_{3}\left(t-1, t-1,1 / \sigma_{1}, 1 / \sigma_{2}, y\right)-\Delta_{3}\left(t-2, t, 1 / \sigma_{1}, 1 / \sigma_{2}, y\right) \\
& -\Delta_{3}\left(t-1, t-1,1 / \sigma_{2}, 1 / \sigma_{1}, y\right)+\Delta_{3}\left(t-2, t, 1 / \sigma_{2}, 1 / \sigma_{1}, y\right) .
\end{aligned}
$$

$$
\begin{aligned}
F_{z}(x)=K_{c c} & \left\{\int_{0}^{\infty} e^{-\lambda_{2} / \sigma_{2}} \lambda_{2}^{t-2}\left(\lambda_{2} \int_{\lambda_{2}}^{x \lambda_{2}} u^{t-2} e^{-u / \sigma_{1}} d u-\int_{\lambda_{2}}^{x \lambda_{2}} u^{t-1} e^{-u / \sigma_{1}} d u\right) d \lambda_{2}\right. \\
& \left.-\int_{0}^{\infty} e^{-\lambda_{2} / \sigma_{1}} \lambda_{2}^{t-2}\left(\lambda_{2} \int_{\lambda_{2}}^{x \lambda_{2}} u^{t-2} e^{-u / \sigma_{2}} d u-\int_{\lambda_{2}}^{x \lambda_{2}} u^{t-1} e^{-u / \sigma_{2}} d u\right) d \lambda_{2}\right\} .
\end{aligned}
$$

$$
\begin{aligned}
f(z)=K_{c c} & \left(\Delta_{4}\left(t-1, t-1,1 / \sigma_{1}, 1 / \sigma_{2}, z\right)-\Delta_{4}\left(t-2, t, 1 / \sigma_{1}, 1 / \sigma_{2}, z\right)\right. \\
& \left.-\Delta_{4}\left(t-1, t-1,1 / \sigma_{2}, 1 / \sigma_{1}, z\right)+\Delta_{4}\left(t-2, t, 1 / \sigma_{2}, 1 / \sigma_{1}, z\right)\right), \quad z \geq 1
\end{aligned}
$$


where $\boldsymbol{\sigma}_{[\ell]}$ is the set of eigenvalues of $\boldsymbol{\Sigma}_{s}$ with the $\ell$-th eigenvalue removed, and $g_{s-1, t}\left(\lambda_{1}, \sigma_{[\ell]}\right)$ denotes the density of the maximum eigenvalue of a reduced-dimension semicorrelated Wishart matrix $\mathbf{W} \stackrel{\mathrm{d}}{\sim} \mathcal{C W}_{s-1}\left(t, \operatorname{diag}\left(\boldsymbol{\sigma}_{[\ell]}\right)\right)$. The constant, $\mathcal{K}_{\ell}$, is given by

$$
\mathcal{K}_{\ell}=\frac{(-1)^{\ell(\ell-1) / 2}}{(t-s+1) !} \sigma_{\ell}^{s-t-1} \prod_{\substack{i=1 \\ i \neq \ell}}^{s} \frac{\sigma_{i}}{\sigma_{i}-\sigma_{\ell}} .
$$

In addition, the density $f\left(\lambda_{1}, \lambda_{s}\right)$ is bounded above by $f\left(\lambda_{1}, \lambda_{s}\right) \leq f_{\text {asy }}\left(\lambda_{1}, \lambda_{s}\right)$.

\section{Proof: See Appendix B.}

We can now present the main theorem for the asymptotic SCN complementary CDF (CCDF).

Theorem 4: Let $z$ denote the $\mathrm{SCN}$ of $\mathbf{W} \stackrel{\mathrm{d}}{\sim} \mathcal{C W}_{s}\left(t, \boldsymbol{\Sigma}_{s}\right)$. Then, as $x$ tends to infinity

$\operatorname{Pr}(z>x) \sim p_{\text {asy }}(x)=\left(\sum_{\ell=1}^{s} \mathcal{K}_{\ell} \mu_{\ell}\right) x^{-(t-s+1)}, \quad(x \rightarrow \infty)$

where $\mu_{\ell}$ denotes the $(t-s+1)$-th moment of the maximum eigenvalue of a reduced-dimension semi-correlated Wishart matrix $\mathbf{W} \stackrel{\text { d }}{\sim} \mathcal{C} \mathcal{W}_{s-1}\left(t, \operatorname{diag}\left(\sigma_{[\ell]}\right)\right)$, i.e.

$$
\mu_{\ell}=\int_{0}^{\infty} \lambda_{1}^{t-s+1} g_{s-1, t}\left(\lambda_{1}, \boldsymbol{\sigma}_{[\ell]}\right) d \lambda_{1} .
$$

Moreover, we have that $\operatorname{Pr}(z>x) \leq p_{\text {asy }}(x)$.

\section{Proof: See Appendix C.}

A closed-form expression for $\mu_{\ell}$, which relies on the marginal eigenvalue statistics of central Wishart matrices, can be found in [24, Eq. (23)]; therefore, Theorem 4 represents a new closed-form solution for the asymptotic tail distribution of semi-correlated central Wishart matrices of arbitrary dimension. Combined with Lemma 1, this result also provides a closed-form approximation for the high-tail distribution of complex non-central Wishart matrices.

To gain more insights, it is convenient to consider the case of dual Wishart matrices, for which the asymptotic tail distributions admit particularly simple forms. These are given in the following two corollaries, for the case of central uncorrelated and semi-correlated Wishart matrices, respectively.

Corollary 3: Let $z$ denote the SCN of $\mathbf{W} \stackrel{\text { d }}{\sim} \mathcal{C W}_{2}\left(t, \mathbf{I}_{2}\right)$. Then, as $x$ tends to infinity,

$$
\operatorname{Pr}(z>x) \sim \frac{(2 t-1) !}{((t-1) !)^{2} x^{t-1}}, \quad(x \rightarrow \infty) .
$$

Proof: Obtained by taking $z$ large in (15) and integrating.

Note that an analogous expression has also been derived in [44, Page 13]. In the context of MIMO communications, this result shows that by increasing the number of antennas, $t$, we can reduce the probability of experiencing an ill-conditioned channel in an uncorrelated Rayleigh fading environment.

Corollary 4: Let $z$ denote the SCN of $\mathbf{W} \stackrel{\mathrm{d}}{\sim} \mathcal{C W}_{2}\left(t, \boldsymbol{\Sigma}_{2}\right)$.
Then, as $x$ tends to infinity,

$$
\operatorname{Pr}(z>x) \sim \frac{(2 t-2) !}{((t-1) !)^{2}} \frac{\left(\sigma_{2}^{2 t-1}-\sigma_{1}^{2 t-1}\right)}{\left|\boldsymbol{\Sigma}_{2}\right|^{t-1}\left(\sigma_{2}-\sigma_{1}\right) x^{t-1}}, \quad(x \rightarrow \infty) .
$$

Proof: Obtained by taking $z$ large in (19) and integrating.

This result shows that the presence of spatial correlation tends to increase the probability of experiencing a highly illconditioned channel. To see this, note that the determinant in the denominator of (25) varies inversely with the level of correlation, satisfying $0 \leq\left|\boldsymbol{\Sigma}_{2}\right| \leq 1$, with the right-hand side equality holding when the channel is uncorrelated (i.e. $\left.\boldsymbol{\Sigma}_{2}=\mathbf{I}_{2}\right)$. Moreover, the ratio $\left(\sigma_{2}^{2 t-1}-\sigma_{1}^{2 t-1}\right) /\left(\sigma_{2}-\sigma_{1}\right)$ varies monotonically with the level of correlation, attaining its minimal value, $2 t-1$, when $\sigma_{1}=\sigma_{2}=1$.

For all results in this section, we have shown that the probability of drawing a highly ill-conditioned channel goes to zero polynomially as $x^{-(t-s+1)}$. This implies that the conditioning of the channel is significantly improved for highly "rectangular" matrices (i.e. $t \gg s$ ), compared with "square" Wishart matrices (i.e. $t=s$ ). To further investigate this phenomenon, we note that the upper tail behavior is intimately related to the existence of moments of the distribution.

The existence of the moments for both central uncorrelated and semi-correlated Wishart matrices is given by the following theorem:

Theorem 5: Let $z$ denote the SCN of $\mathbf{W} \stackrel{\mathrm{d}}{\sim} \mathcal{C W}_{s}\left(t, \mathbf{I}_{s}\right)$ or $\mathbf{W} \stackrel{\mathrm{d}}{\sim} \mathcal{C W}_{s}\left(t, \boldsymbol{\Sigma}_{s}\right)$. Then, only the first $t-s$ moments of $z$ exist.

Proof: See Appendix D.

This property helps to explain the badly behaved nature of the condition number distributions, especially when $t \approx s$. For example, when $t=s$, the mean does not exist; for $t=s+1$, the mean does exist but not the variance; for $t=s+2$, the variance exists but no higher order moments, and so forth.

\section{Statistics of the Demmel Condition Number}

The DCN, defined as

$$
\kappa=\frac{\eta_{s}}{\lambda_{s}}, \quad \kappa \geq s
$$

with $\eta_{s}=\sum_{k=1}^{s} \lambda_{k}$ denoting the matrix trace, is another important metric which has been considered in various areas, such as numerical analysis, sphericity tests in multivariate statistics, eigenvalue computation, and matrix inversion. For a detailed review of DCN mathematical applications, see [57]. Apart from $[39,40]$, the DCN has also found application in the analysis of indoor MIMO-OFDM systems [58], and in the analytical prediction of level-crossing and fade duration statistics of i.i.d. Rayleigh channels [59]. We now derive new expressions for the distribution of the DCN for the channel models introduced in Section II.

\section{A. Exact Expressions for the DCN of Dual Wishart Matrices}

In general, it appears quite difficult to obtain an exact characterization of the DCN statistics for arbitrary dimensions, as was presented for the SCN in Theorem 1. The main 
challenge is the complexity of the required multi-dimensional integration in this case, which, for matrix dimensions $s>2$, needs to be performed over a complicated surface. For more details, see [60, Eq. (24)] and the subsequent discussion, which tackled a similar problem in the context of radar signal processing.

For this reason, we explicitly focus on the case of dual Wishart matrices. We start by presenting a new expression for the CDF and PDF of the DCN for the case of dual uncorrelated central Wishart matrices, and then consider the more general case of semi-correlated central Wishart. We note that alternative DCN expressions can be directly derived by combining the SCN results of Section III-B with the relationship $\kappa=z+1$, which holds for the dual case. The following analysis, however, follows a slightly different line of reasoning and leads to simplified expressions.

Theorem 6: The PDF of the DCN of $\mathbf{W} \stackrel{\mathrm{d}}{\sim} \mathcal{C W}_{2}\left(t, \mathbf{I}_{2}\right)$ is given by

$$
f(\kappa)=c_{1} \kappa^{-2 t}(\kappa-1)^{t-2}(2-\kappa)^{2}, \quad \kappa \geq 2
$$

where $c_{1}=\Gamma(2 t) /(\Gamma(t) \Gamma(t-1))$, and $\Gamma(\cdot)$ denotes the gamma function.

Proof: The proof stems from a result presented recently in $[60$, Eq. (25)], which returns the PDF of the ratio of the maximum eigenvalue to the matrix trace, i.e. $\beta=\lambda_{1} / \eta_{s}$. Due to symmetry, this also holds for the inverse of the DCN, $\zeta=$ $1 / \kappa$, with the only difference pertaining to the interval bounds, i.e. $0 \leq \zeta \leq 1 / 2$. Thus, by applying a transformation of variables, the result is obtained.

Corollary 5: The CDF of the DCN of $\mathbf{W} \stackrel{\mathrm{d}}{\sim} \mathcal{C W}_{2}\left(t, \mathbf{I}_{2}\right)$ is given by

$$
F_{\kappa}(x)=c_{1}\left(\Delta_{5}(t, x)-\Delta_{5}(t, 2)\right)
$$

where

$$
\begin{aligned}
& \Delta_{5}(n, y)=\sum_{k=0}^{n-2}\left(\begin{array}{c}
n-2 \\
k
\end{array}\right)(-1)^{k+1} \\
& \quad \times\left(\frac{4 y^{-(n+k+1)}}{n+k+1}-\frac{4 y^{-(n+k)}}{n+k}+\frac{4 y^{-(n+k-1)}}{n+k-1}\right) .
\end{aligned}
$$

Proof: Referring back to (27), the CDF is equal to $F_{\kappa}(x)=\int_{2}^{x} f(\kappa) d \kappa$ which upon applying a binomial expansion and evaluating all integrals term-by-term, yields (28).

Theorem 7: The PDF of the DCN of $\mathbf{W} \stackrel{\mathrm{d}}{\sim} \mathcal{C W}_{2}\left(t, \boldsymbol{\Sigma}_{2}\right)$ is given by

$$
\begin{aligned}
& f(\kappa)=c_{2}(\kappa-2)(\kappa-1)^{t-2} \\
& \times\left(\Delta_{6}\left(\sigma_{1}, \sigma_{2}, \kappa\right)^{1-2 t}-\Delta_{6}\left(\sigma_{2}, \sigma_{1}, \kappa\right)^{1-2 t}\right), \quad \kappa \geq 2
\end{aligned}
$$

where $c_{2}=\Gamma(2 t-1)\left(\sigma_{1} \sigma_{2}\right)^{1-t} /\left(\Gamma(t) \Gamma(t-1)\left(\sigma_{1}-\sigma_{2}\right)\right)$, and

$$
\Delta_{6}(a, b, y)=\frac{y-1}{a}+\frac{1}{b} \text {. }
$$

Proof: We begin with the joint eigenvalue PDF of a dual semi-correlated central Wishart matrix

$$
\begin{aligned}
f\left(\lambda_{1}, \lambda_{2}\right) & =K_{c c}\left(\lambda_{2}-\lambda_{1}\right)\left(\lambda_{1} \lambda_{2}\right)^{t-2} \\
& \times\left(e^{-\left(\lambda_{1} / \sigma_{1}+\lambda_{2} / \sigma_{2}\right)}-e^{-\left(\lambda_{2} / \sigma_{1}+\lambda_{1} / \sigma_{2}\right)}\right) .
\end{aligned}
$$

Setting $\eta_{2}=\lambda_{1}+\lambda_{2}$ and $\zeta=\lambda_{2} / \eta_{2}$, we apply the transformation: $\left(\lambda_{1}, \lambda_{2}\right) \mapsto\left(\eta_{2}, \zeta\right)$. Integrating out $\eta_{2}$ using the integral identity [53, Eq. (3.351.3)] and after some algebraic manipulations and simplifications we can obtain the desired result.

Corollary 6: The CDF of the DCN of $\mathbf{W} \stackrel{\mathrm{d}}{\sim} \mathcal{C W}_{2}\left(t, \boldsymbol{\Sigma}_{s}\right)$ is given by (33) at the bottom of the page where

$$
\begin{gathered}
\Delta_{7}(a, b, \nu, y)=\left(\frac{\nu}{a}\right)^{2 t-1} \sum_{k=0}^{t-2}\left(\begin{array}{c}
t-2 \\
k
\end{array}\right)(-1)^{k} \\
\times\left(I_{1}(t-k-1, b, a, 2 t-1, x)\right. \\
\left.\quad-2 I_{1}(t-k-2, b, a, 2 t-1, x)\right)
\end{gathered}
$$

with

$$
I_{1}(n, b, a, m, y)=\frac{y^{n+1}}{n+1}{ }_{2} F_{1}(m, n+1 ; n+2 ;-y b / a)
$$

where ${ }_{2} F_{1}(\cdot, \cdot ; \cdot ; \cdot)$ denotes the classical Gaussian hypergeometric function [53, Eq. (9.14.2)].

Proof: The result is obtained by substituting (30) in $F_{\kappa}(x)=\int_{2}^{x} f(\kappa) d \kappa$, applying the binomial expansion and solving the resulting integrals using [53, Eq. (3.194)].

As before, the case of dual uncorrelated non-central Wishart matrices can be tackled via Theorem 7 and Corollary 6 respectively, upon application of Lemma 1. Finally, for the case of dual Wishart matrices, the DCN limiting distributions (for asymptotically large values) are identical to those of the $\mathrm{SCN}$, as given in Corollary 3 and Corollary 4 for central uncorrelated and semi-correlated Wishart matrices respectively. This follows since $\kappa=z+1$ and in the high-tail $z \gg 1$.

\section{Numerical Results}

In this section, we validate the theoretical analysis presented in Sections III and IV through comparison with MonteCarlo simulations. We also investigate the implications of the model parameters on the condition number distributions. The simulation results are obtained by generating 50,000 random samples of the random matrix $\mathbf{H}$ according to (2)-(4), for the three different Wishart scenarios under investigation. For

$$
\begin{aligned}
F_{\kappa}(x)=c_{2} & \left(\Delta_{7}\left(\frac{\sigma_{1}-\sigma_{2}}{\sigma_{1}}, \frac{\sigma_{2}}{\sigma_{1}}, \sigma_{2}, x\right)-\Delta_{7}\left(\frac{\sigma_{2}-\sigma_{1}}{\sigma_{2}}, \frac{\sigma_{1}}{\sigma_{2}}, \sigma_{1}, x\right)\right. \\
& \left.-\Delta_{7}\left(\frac{\sigma_{1}-\sigma_{2}}{\sigma_{1}}, \frac{\sigma_{2}}{\sigma_{1}}, \sigma_{2}, 2\right)+\Delta_{7}\left(\frac{\sigma_{2}-\sigma_{1}}{\sigma_{2}}, \frac{\sigma_{1}}{\sigma_{2}}, \sigma_{1}, 2\right)\right), \quad x \geq 2
\end{aligned}
$$




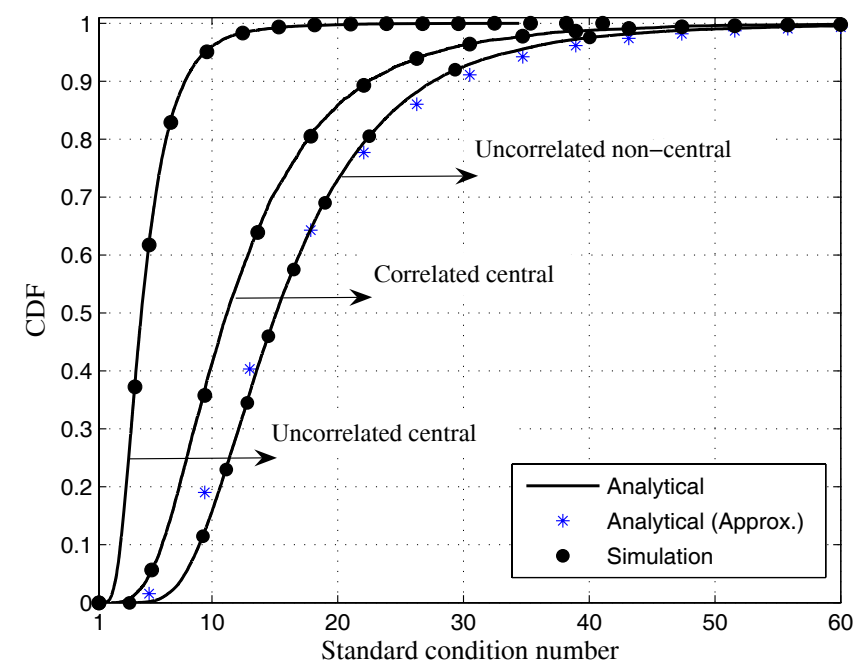

Fig. 1. SCN CDF of an $8 \times 3$ MIMO channel under uncorrelated Rayleigh, semi-correlated Rayleigh $(\rho=0.6)$ and uncorrelated Ricean $\left(K_{r}=3 \mathrm{~dB}\right)$ fading.

the semi-correlated Wishart case, we construct $\boldsymbol{\Sigma}_{s}$ according to the widely used exponential correlation model [61], due to its simplicity. Specifically, the entries of $\boldsymbol{\Sigma}_{s}$ are generated as $\left(\boldsymbol{\Sigma}_{s}\right)_{i, j}=\rho^{|i-j|}$, where $\rho \in[0,1)$. For the uncorrelated non-central Wishart case, we model the deterministic channel component (channel mean) using the conventional configuration [48], which returns a single dominant eigenvalue. It is important to note, however, that our analytical results apply to arbitrary correlation structures (with distinct eigenvalues), and to LoS MIMO configurations with arbitrary rank of the mean channel matrix.

Figure 1 validates the generic framework for the SCN CDF, presented in Theorem 1 . An $8 \times 3$ MIMO channel is considered under all three different fading conditions: uncorrelated, semicorrelated Rayleigh (with $\rho=0.6$ ), and uncorrelated Ricean (with $K_{r}=3 \mathrm{~dB}$ ). For the latter case, we consider both the infinite series expansion introduced in (12) (truncated to the first thirty dominant terms) as well as the simplified approximation based on Lemma 1. We see that the analytical curves for all cases agree precisely with the simulations, and that the analytical central-non-central Wishart approximation is accurate. Moreover, the presence of either spatial correlation or non-zero mean tends to increase the SCN spread and shifts the corresponding $\mathrm{CDF}$ to the right, thereby degrading the channel conditioning.

Figure 2 investigates the asymptotic high-tail behavior of the SCN distribution under semi-correlated Rayleigh fading. Results are shown for the same MIMO configuration as in Fig. 1, for various values of $\rho$. The simulated curves represent the scaled probability, $x^{t-s+1} \operatorname{Pr}(z>x)$, and are clearly seen to converge to the analytical limiting values predicted by Theorem 4. Moreover, we see that higher values of $\rho$ tend to increase the probability of experiencing an ill-conditioned channel, whilst also reducing the convergence speed of the associated curves.

Figure 3 verifies the closed-form expressions for the CDF and PDF of the DCN of dual central uncorrelated Wishart matrices, given in Corollary 5 and Theorem 6 respectively. Re-

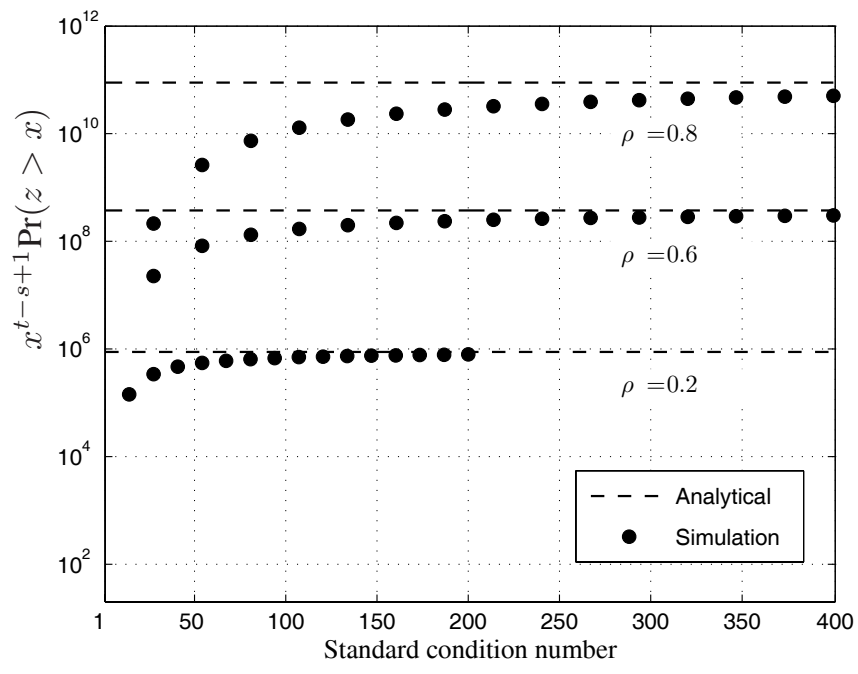

Fig. 2. High-tail behavior of SCN CDF of an $8 \times 3$ MIMO channel under semi-correlated Rayleigh fading.

sults are shown for $N_{t}=2$ and various $N_{r}$. As expected, there is a precise match between the analysis and simulations. We clearly see that the conditioning of the channel significantly improves as $N_{r}$ increases (i.e. the channel matrix becomes "more rectangular").

Figure 4 more closely investigates the effect of spatial correlation on the SCN and DCN distributions. The analytical results are based on Theorem 3 and Corollary 6, respectively. Both figures confirm that the presence of spatial correlation leads to a significant reduction in the conditioning of the channel. Note also that the corresponding SCN and DCN curves (i.e. for a given $\rho$ ) are very similar. This is explained by recalling that for the dual case under consideration the following relationship holds $\kappa=z+1$.

Finally, the effect of the $K$-factor on the SCN is depicted in Fig. 5. The analytical results are based on Corollary 2, and the simplified approximation in Lemma 1. We see that in all cases, the analytical approximation yields good accuracy. Moreover, as $K_{r}$ increases, the mean and the variance of $\mathrm{SCN}$ increase as well, demonstrating that the conditioning of the channel degrades with $K_{r}$. Note however, that this behavior is due to the assumption that the channel mean has a single dominant eigenvalue; consequently, as $K_{r} \rightarrow \infty$, the SCN becomes infinitely large. In contrast, for more well conditioned LoS configurations, the effect of $K_{r}$ may not be so severe.

\section{CONCLUSION}

The statistical properties of the SCN of complex Wishart matrices have key importance in the field of MIMO systems, as well as various other applications. In this contribution, we have presented a new generic framework for characterizing the SCN distribution of complex Wishart matrices, which yields a simple unified expression applying for both central and non-central Wishart distributions of arbitrary dimension. For the special and practically important case of dual Wishart matrices, we also derived exact polynomial expressions for the $\mathrm{SCN}$ and DCN distributions.

In addition, we proposed a novel closed-form expression for the asymptotic tail distribution of the $\mathrm{SCN}$ for correlated 


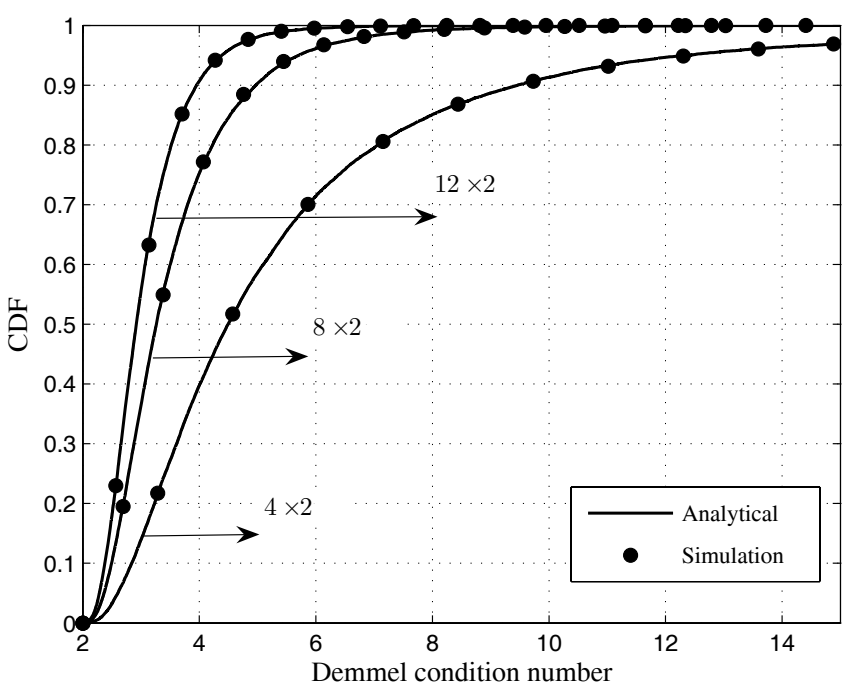

(a) CDF of Demmel condition number.

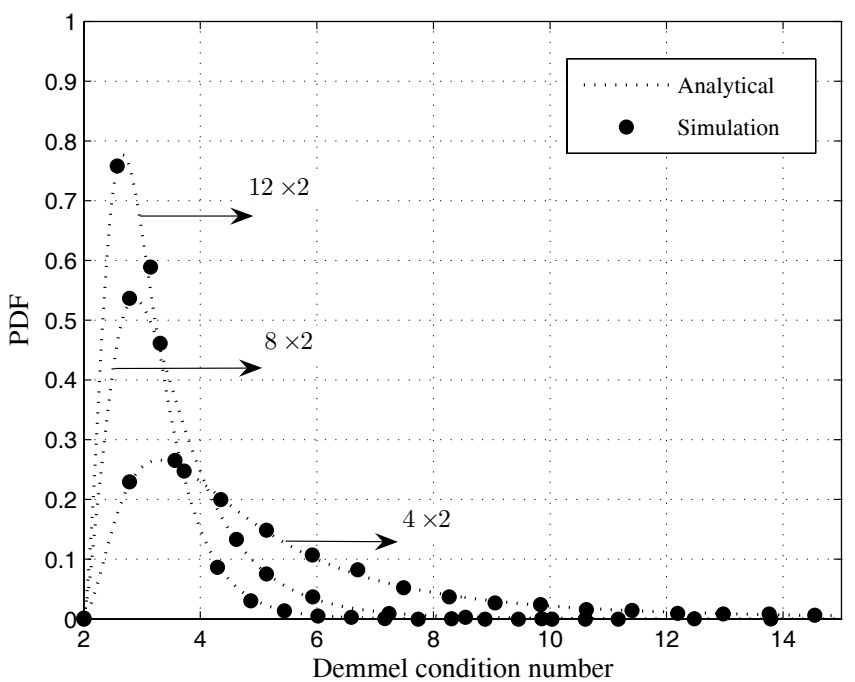

(b) PDF of Demmel condition number.

Fig. 3. DCN CDF and PDF of a dual uncorrelated Rayleigh MIMO channel for different numbers of receive antennas.

central Wishart matrices of arbitrary dimension, and demonstrated an interesting relationship to the maximum eigenvalue moments of Wishart matrices of smaller dimension. Our analytical results were verified through comparison with MonteCarlo simulations and were used to gain fundamental insights of the statistical behavior of the channel conditioning for various MIMO fading scenarios, such as uncorrelated/semicorrelated Rayleigh fading and Ricean fading.

\section{APPENDIX A}

\section{Proof OF THEOREM 1}

The CDF of the SCN can be evaluated in an integral form as follows

$$
F_{z}(x)=\int_{0}^{\infty}\left[\int_{\lambda_{2}}^{x \lambda_{s}} \cdots \int_{\lambda_{s}}^{x \lambda_{s}} f(\boldsymbol{\lambda}) d \lambda_{s-1} \ldots d \lambda_{1}\right] d \lambda_{s}
$$

We emphasize the fact that the multiple integral term inside the square brackets of (36) denotes the probability that $\left(\lambda_{1}, \lambda_{2}, \ldots, \lambda_{s-1}\right)$ all lie within the interval $\left[\lambda_{s}, x \lambda_{s}\right]$. Since

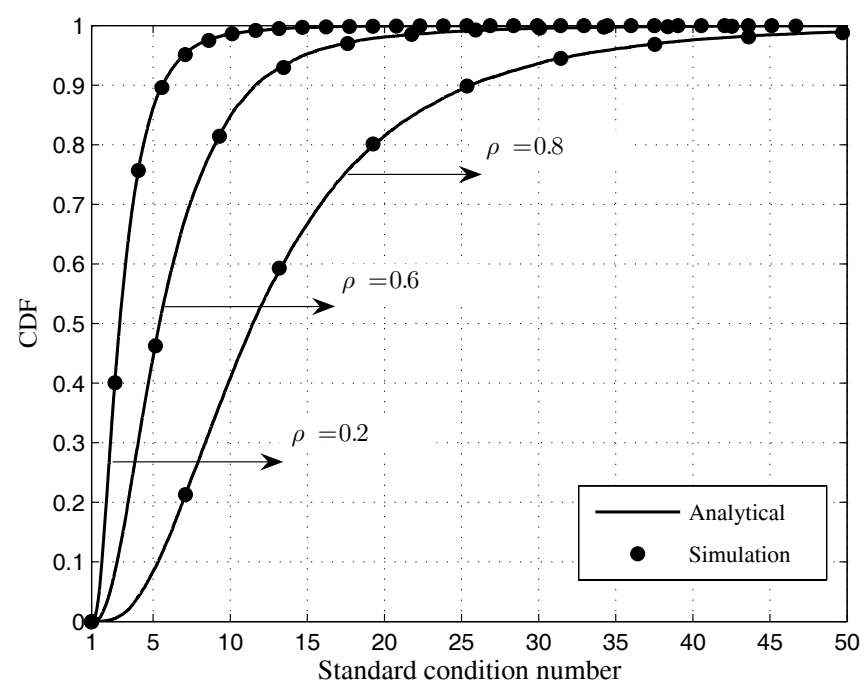

(a) $\mathrm{CDF}$ of standard condition number.

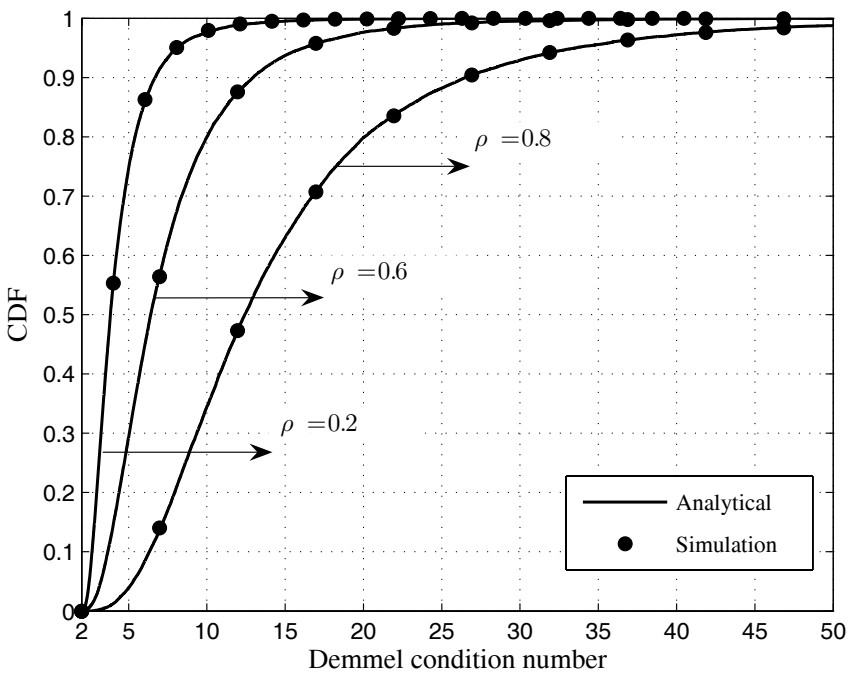

(b) $\mathrm{CDF}$ of Demmel condition number.

Fig. 4. SCN and DCN CDF of a $6 \times 2$ semi-correlated Rayleigh MIMO channel for different correlation coefficients, $\rho$.

$f(\boldsymbol{\lambda})$ is symmetric in all of its arguments, we can reformulate (36) as

$F_{z}(x)=\frac{1}{(s-1) !} \int_{0}^{\infty} \int_{\lambda_{s}}^{x \lambda_{s}} \cdots \int_{\lambda_{s}}^{x \lambda_{s}} f(\boldsymbol{\lambda}) d \lambda_{1} \ldots d \lambda_{s-1} d \lambda_{s}$.

Now, substituting (6) into (37) and expanding $|\boldsymbol{\Phi}(\boldsymbol{\lambda})|$ using the definition of the determinant (i.e. Liebniz determinant formula), we obtain

$$
\begin{array}{r}
F_{z}(x)=\frac{K}{(s-1) !} \int_{0}^{\infty} \sum_{\alpha}(-1)^{\alpha} \int_{\lambda_{s}}^{x \lambda_{s}} \ldots \int_{\lambda_{s}}^{x \lambda_{s}} \mid \phi_{\alpha_{i}}\left(\lambda_{i}\right) \\
\times \psi_{j}\left(\lambda_{i}\right) \xi\left(\lambda_{i}\right) \mid d \lambda_{1} \ldots d \lambda_{s-1} d \lambda_{s}
\end{array}
$$

where $\alpha=\left\{\alpha_{1}, \alpha_{2}, \ldots, \alpha_{s}\right\}$ is a permutation of the integers $\{1,2, \ldots, s\}$, and the summation is taken over all such permutations. This can be further simplified as

$$
F_{z}(x)=\frac{K}{(s-1) !} \int_{0}^{\infty} \sum_{\alpha}(-1)^{\alpha}\left|I_{\alpha_{i}, j}\right| d \lambda_{s}
$$




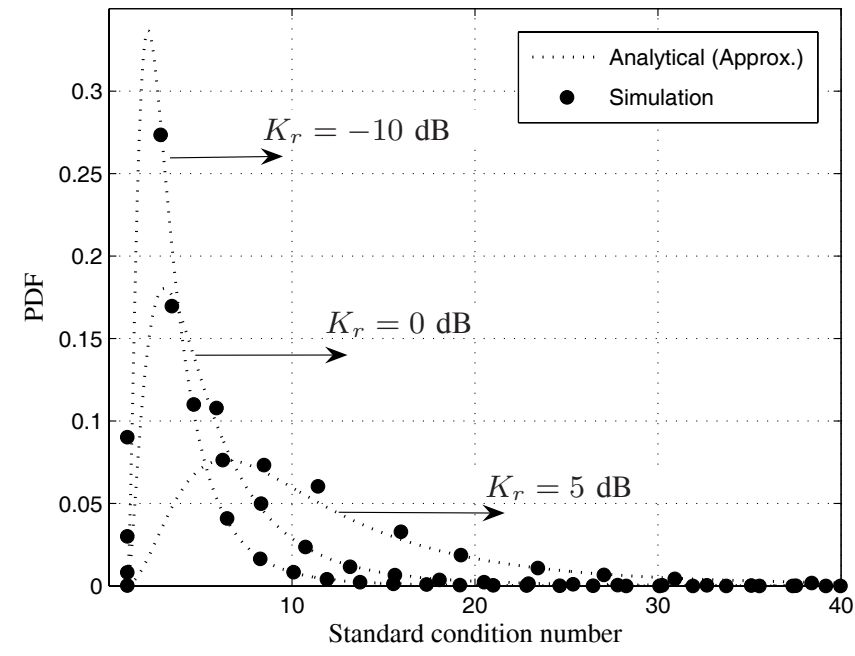

Fig. 5. SCN PDF of a $2 \times 5$ uncorrelated Ricean MIMO channel for different Ricean $K$-factors, $K_{r}$.

where the elements of the matrix $I_{a_{i}, j},(1 \leq i, j \leq s)$, are given by

$$
I_{\alpha_{i}, j}= \begin{cases}\int_{\lambda_{s}}^{x \lambda_{s}} \phi_{\alpha_{i}}(u) \psi_{j}(u) \xi(u) d u, & \text { if } i \neq s \\ \phi_{\alpha_{i}}\left(\lambda_{s}\right) \psi_{j}\left(\lambda_{s}\right) \xi\left(\lambda_{s}\right), & \text { if } i=s .\end{cases}
$$

After reordering the rows, the determinant of $I_{a_{i}, j}$ can be written as

$$
\left|I_{\alpha_{i}, j}\right|=(-1)^{\alpha}\left|\left[\begin{array}{ll}
\int_{\lambda_{s}}^{x \lambda_{s}} \phi_{\alpha_{i}}(u) \psi_{j}(u) \xi(u) d u, & \text { if } i \neq \alpha_{s} \\
\phi_{\alpha_{i}}\left(\lambda_{s}\right) \psi_{j}\left(\lambda_{s}\right) \xi\left(\lambda_{s}\right), & \text { if } i=\alpha_{s}
\end{array}\right]\right|
$$

The result now follows by substituting (41) into (39) and collecting common terms in the summation.

\section{APPENDIX B}

\section{PRoOF OF LEMMA 2}

The joint distribution of the maximum and minimum eigenvalues is calculated by integrating the joint eigenvalue PDF (6) as follows

$$
f\left(\lambda_{1}, \lambda_{s}\right)=\int \cdots \int_{\mathcal{D}} f(\boldsymbol{\lambda}) d \lambda_{s-1} \ldots d \lambda_{2}
$$

where the multiple integration is taken over the region $\mathcal{D}=$ $\left\{\lambda_{s} \leq \lambda_{s-1} \leq \ldots \leq \lambda_{2} \leq \lambda_{1}\right\}$. To address this integration, we first aim to isolate terms involving $\lambda_{s}$ in the joint eigenvalue PDF (6). To this end, first note that

$$
\begin{aligned}
& |\boldsymbol{\Phi}(\boldsymbol{\lambda})|=\left|\lambda_{j}^{i-1}\right|=\prod_{1 \leq i<j \leq s}\left(\lambda_{j}-\lambda_{i}\right) \\
& =(-1)^{s-1} \prod_{1 \leq i<j \leq s-1}\left(\lambda_{j}-\lambda_{i}\right) \prod_{i=1}^{s-1}\left(\lambda_{i}-\lambda_{s}\right)
\end{aligned}
$$

and apply Laplace's expansion to expand the determinant $|\Psi(\lambda)|$ along its last column as

$$
|\boldsymbol{\Psi}(\boldsymbol{\lambda})|=\left|\psi_{i}\left(\lambda_{j}\right)\right|=\sum_{\ell=1}^{s}(-1)^{\ell+s} \psi_{\ell}\left(\lambda_{s}\right)\left|\Psi_{\ell}\left(\lambda_{1}, \ldots, \lambda_{s-1}\right)\right|
$$

where $\boldsymbol{\Psi}_{\ell}\left(\lambda_{1}, \ldots, \lambda_{s-1}\right)$ is the minor of $\boldsymbol{\Psi}(\boldsymbol{\lambda})$ with the last column and $\ell$-th row removed.

Substituting (6) into (42) and using (43) and (44), we obtain

$$
f\left(\lambda_{1}, \lambda_{s}\right)=K \xi\left(\lambda_{s}\right) \sum_{\ell=1}^{s}(-1)^{\ell-1} \psi_{\ell}\left(\lambda_{s}\right) \mathcal{I}_{\ell}\left(\lambda_{1}, \lambda_{s}\right)
$$

where

$$
\begin{gathered}
\mathcal{I}_{\ell}\left(\lambda_{1}, \lambda_{s}\right)=\int \ldots \int_{\mathcal{D}} \prod_{i=1}^{s-1} \xi\left(\lambda_{i}\right) \prod_{1 \leq i<j \leq s-1}\left(\lambda_{j}-\lambda_{i}\right) \\
\quad \times \prod_{i=1}^{s-1}\left(\lambda_{i}-\lambda_{s}\right)\left|\Psi_{\ell}\left(\lambda_{1}, \ldots, \lambda_{s-1}\right)\right| d \lambda_{s-1} \ldots d \lambda_{2} .
\end{gathered}
$$

Now, using similar arguments to [44, Lemma 3.1], we can apply the Dominated Convergence Theorem and observe that as $\lambda_{s} \rightarrow 0$ the integrand converges upwards to a constant which, by comparison with (6), matches precisely with the joint eigenvalue density of a reduced-dimension semicorrelated Wishart matrix $\mathbf{W} \stackrel{\mathrm{d}}{\sim} \mathcal{C} \mathcal{W}_{s-1}\left(t, \operatorname{diag}\left(\boldsymbol{\sigma}_{[\ell]}\right)\right)$, but without the normalization constant $K$. As such, all eigenvalues except for $\lambda_{1}$ are integrated out, and we have

$$
\lim _{\lambda_{1} \rightarrow 0} \mathcal{I}_{\ell}\left(\lambda_{1}, \lambda_{s}\right)=\frac{g_{s-1, t}\left(\lambda_{1}, \boldsymbol{\sigma}_{[\ell]}\right)}{K_{c c}\left(\boldsymbol{\sigma}_{[\ell]}\right)}
$$

with $K_{c c}\left(\boldsymbol{\sigma}_{[\ell]}\right)$ denoting the integration constant $K_{c c}$ for the distribution $\mathbf{W} \stackrel{\mathrm{d}}{\sim} \mathcal{C} \mathcal{W}_{s-1}\left(t, \operatorname{diag}\left(\sigma_{[\ell]}\right)\right)$ (see Table I). Finally, substituting (47) into (45), noting that $\xi\left(\lambda_{s}\right)=\lambda_{s}^{t-s}$ and that

$$
\psi_{\ell}\left(\lambda_{s}\right)=e^{-\lambda_{s} / \sigma_{\ell}}=1+o(1), \quad\left(\lambda_{s} \rightarrow 0\right)
$$

and performing some basic algebraic manipulations yields the desired result.

In order to validate the upper bound for the joint eigenvalue PDF $f\left(\lambda_{1}, \lambda_{s}\right) \leq f_{\text {asy }}\left(\lambda_{1}, \lambda_{s}\right)$, we refer back to (42) and upper bound the joint distribution of the maximum and minimum eigenvalues as follows

$$
f\left(\lambda_{1}, \lambda_{s}\right) \leq \int \ldots \int_{\widetilde{\mathcal{D}}} f(\boldsymbol{\lambda}) d \lambda_{s-1} \ldots d \lambda_{2}
$$

where the integration is performed over the expanded region $\widetilde{\mathcal{D}}=\left\{0 \leq \lambda_{s-1} \leq \ldots \leq \lambda_{2} \leq \lambda_{1}\right\}$. Omitting explicit details for the sake of clarity, the final result is obtained by substituting the joint eigenvalue PDF (6), further upper bounding it via $\prod_{i=1}^{s-1}\left(\lambda_{i}-\lambda_{s}\right) \leq \prod_{i=1}^{s-1} \lambda_{i}$, and then following the same procedure as above.

\section{APPENDIX C \\ PROOF OF THEOREM 4}

To prove (22), we are required to show

$$
\lim _{x \rightarrow \infty} x^{t-s+1} \operatorname{Pr}(z>x)=\sum_{\ell=1}^{s} \mathcal{K}_{\ell} \mu_{\ell} .
$$

The exact tail distribution is given by

$$
\operatorname{Pr}(z>x)=\int_{0}^{\infty} \int_{0}^{x^{-1} \lambda_{1}} f\left(\lambda_{1}, \lambda_{s}\right) d \lambda_{s} d \lambda_{1} .
$$


Therefore, let us write

$$
\lim _{x \rightarrow \infty} x^{t-s+1} \operatorname{Pr}(z>x)=\lim _{x \rightarrow \infty} \int_{0}^{\infty} \mathcal{J}\left(\lambda_{1}, x\right) d \lambda_{1}
$$

where

$$
\mathcal{J}\left(\lambda_{1}, x\right)=x^{t-s+1} \int_{0}^{x^{-1} \lambda_{1}} f\left(\lambda_{1}, \lambda_{s}\right) d \lambda_{s}
$$

From (20), since

$$
\begin{aligned}
& f\left(\lambda_{1}, \lambda_{s}\right)=(t-s+1) \lambda_{s}^{t-s} \\
& \quad \times \sum_{\ell=1}^{s} \mathcal{K}_{\ell} g_{s-1, t}\left(\lambda_{1}, \boldsymbol{\sigma}_{[\ell]}\right)+o\left(\lambda_{s}^{t-s}\right)
\end{aligned}
$$

as $\lambda_{s} \rightarrow 0$, we see that $\mathcal{J}\left(\lambda_{1}, x\right)$ converges pointwise to

$$
\lim _{x \rightarrow \infty} \mathcal{J}\left(\lambda_{1}, x\right)=\lambda_{1}^{t-s+1} \sum_{\ell=1}^{s} \mathcal{K}_{\ell} g_{s-1, t}\left(\lambda_{1}, \boldsymbol{\sigma}_{[\ell]}\right) .
$$

We now show that the limit and integral in (52) can be exchanged. To this end, recall from Lemma 2 that $f_{\text {asy }}\left(\lambda_{1}, \lambda_{s}\right)$, the asymptotic expression for $f\left(\lambda_{1}, \lambda_{s}\right)$, is also an upper bound (and thus dominates) the exact expression for $f\left(\lambda_{1}, \lambda_{s}\right)$. Therefore, by substituting the right-hand side of (20) into (53) (i.e., substituting the expression for $f_{\text {asy }}\left(\lambda_{1}, \lambda_{s}\right)$ in place of $f\left(\lambda_{1}, \lambda_{s}\right)$ ), we can immediately obtain an upper bound on $\mathcal{J}\left(\lambda_{1}, x\right)$, and this bound evaluates to the right-hand side of (55).

It then follows that the limiting expression for $\mathcal{J}\left(\lambda_{1}, x\right)$ in (55) (which is clearly independent of $x$ ), serves also as an upper bound for $\mathcal{J}\left(\lambda_{1}, x\right)$ for all $x$. Since this upper bound is integrable with respect to $\lambda_{1}$ over the range $[0, \infty]$, the Dominated Convergence Theorem can be applied to exchange the limit and integral in (52). As a consequence, (52) evaluates to

$$
\begin{aligned}
& \lim _{x \rightarrow \infty} x^{t-s+1} \operatorname{Pr}(z>x) \\
& =\sum_{\ell=1}^{s} \mathcal{K}_{\ell} \int_{0}^{\infty} \lambda_{1}^{t-s+1} g_{s-1, t}\left(\lambda_{1}, \boldsymbol{\sigma}_{[\ell]}\right) d \lambda_{1}
\end{aligned}
$$

which is precisely (50).

Finally, the upper bound $\operatorname{Pr}(z>x) \leq p_{\text {asy }}(x)$ follows by simply substituting the upper bound for $f\left(\lambda_{1}, \lambda_{s}\right)$, as given previously in Lemma 2, into (51) and performing the double integration.

\section{APPENDIX D \\ PROOF OF THEOREM 5}

Given that $z^{r}$ contains the term $\lambda_{1}^{r} \lambda_{s}^{-r}$, the $r$-th moment, $E\left(z^{r}\right)$, of the SCN contains the integral

$$
I_{r}=\int_{0}^{\infty} \int_{0}^{\lambda_{1}} \ldots \int_{0}^{\lambda_{s-1}} \lambda_{1}^{r} \lambda_{s}^{-r} f(\boldsymbol{\lambda}) d \lambda_{s} \ldots d \lambda_{1} .
$$

Combining (6) and Table I, we can write the joint eigenvalue PDF for the uncorrelated and semi-correlated central cases in the following form

$$
f(\boldsymbol{\lambda})=\sum_{i} b_{i} \prod_{j=1}^{s}\left(\lambda_{j}^{t-s+c_{i j}} e^{-\lambda_{j} d_{i j}}\right), \quad 1 \leq i, j \leq s
$$

where $b_{i}, c_{i j}, d_{i j}$ are constants and $c_{i j}, d_{i j} \geq 0$. Clearly, the smallest power of $\lambda_{s}$ in $f(\boldsymbol{\lambda})$ is $\lambda_{s}^{t-s}$, so that $I_{r}$ contains the integral

$$
\int_{0}^{\lambda_{s-1}} \lambda_{s}^{t-s-r} e^{-\lambda_{s} d_{i s}} d \lambda_{s}
$$

The proof follows trivially after noticing that the above integral does not exist for $r>t-s$.

\section{ACKNOWLEDGMENTS}

The authors would like to thank the Associate Editor as well as the anonymous reviewers for their constructive comments which helped to improve the quality of this manuscript.

\section{REFERENCES}

[1] I. E. Telatar, "Capacity of multi-antenna Gaussian channels," Europ. Trans. Telecommun., vol. 10, no. 6, pp. 585-595, Nov./Dec. 1999.

[2] M. Chiani, M. Z. Win, and A. Zanella, "On the capacity of spatially correlated MIMO Rayleigh-fading channels," IEEE Trans. Inf. Theory, vol. 49, no. 10, pp. 2363-2371, Oct. 2003.

[3] H. Shin, M. Z. Win, J. H. Lee, and M. Chiani, "On the capacity of doubly correlated MIMO channels," IEEE Trans. Wireless Commun., vol. 5, no. 8, pp. 2253-2265, Aug. 2006.

[4] M. R. McKay and I. B. Collings, "General capacity bounds for spatially correlated Rician MIMO channels," IEEE Trans. Inf. Theory, vol. 51, no. 9, pp. 3121-3145, Sep. 2005.

[5] M. Matthaiou, Y. Kopsinis, D. I. Laurenson, and A. M. Sayeed, "Upper bound for the ergodic capacity of dual MIMO Ricean systems: simplified derivation and asymptotic tightness," IEEE Trans. Commun., vol. 57, no. 12, pp. 3589-3596, Dec. 2009.

[6] M. Kang and M.-S. Alouini, "Capacity of MIMO Rician channels," IEEE Trans. Wireless Commun., vol. 5, no. 1, pp. 112-122, Jan. 2006.

[7] A. Maaref and S. Aïssa, "Joint and marginal eigenvalue distributions of (non)central complex Wishart matrices and PDF-based approach for characterizing the capacity statistics of MIMO Ricean and Rayleigh fading channels," IEEE Trans. Wireless Commun., vol. 6, no. 10, pp. 3607-3619, Oct. 2007.

[8] P. J. Smith, S. Roy, and M. Shafi, "Capacity of MIMO systems with semicorrelated flat fading," IEEE Trans. Inf. Theory, vol. 49, no. 10, pp. 2781-2788, Oct. 2003.

[9] A. M. Tulino, A. Lozano, and S. Verdú, "Impact of antenna correlation on the capacity of multiantenna channels," IEEE Trans. Inf. Theory, vol. 51, no. 7, pp. 2491-2509, July 2005.

[10] A. Lozano, A. M. Tulino, and S. Verdú, "High-SNR power offset in multiantenna communication," IEEE Trans. Inf. Theory, vol. 51, no. 12, pp. 4134-4151, Dec. 2005.

[11] H. Shin and J. H. Lee, "Capacity of multiple-antenna fading channels: spatial fading correlation, double scattering, and keyhole," IEEE Trans. Inf. Theory, vol. 49, no. 10, pp. 2636-2647, Oct. 2003.

[12] Ö. Oyman, R. U. Nabar, H. Bölcskei, and A. J. Paulraj, "Characterizing the statistical properties of mutual information in MIMO channels," IEEE Trans. Signal Process., vol. 51, no. 11, pp. 2784-2795, Nov. 2003.

[13] G. Alfano, A. Lozano, A. M. Tulino, and S. Verdú, "Mutual information and eigenvalue distribution of MIMO Ricean channels," in Proc. Int. Symp. Inf. Theory Applic. (ISITA), Parma, Italy, Oct. 2004.

[14] M. Kiessling and J. Speidel, "Mutual information of MIMO channels in correlated Rayleigh fading environments - a general solution," in Proc. IEEE Int. Conf. Commun. (ICC), Paris, France, June 2004, pp. 814-818.

[15] X. W. Cui, Q. T. Zhang, and Z. M. Feng, "Generic procedure for tightly bounding the capacity of MIMO correlated Rician fading channels," IEEE Trans. Commun., vol. 53, no. 5, pp. 890-898, May 2005.

[16] P. A. Dighe, R. K. Mallik, and S. S. Jamuar, "Analysis of transmitreceive diversity in Rayleigh fading," IEEE Trans. Commun., vol. 51, no. 4, pp. 694-703, Apr. 2003.

[17] M. R. McKay, A. J. Grant, and I. B. Collings, "Performance analysis of MIMO-MRC in double-correlated Rayleigh environments," IEEE Trans. Commun., vol. 55, no. 3, pp. 497-507, Mar. 2007.

[18] M. Kang and M.-S. Alouini, "Largest eigenvalue of complex Wishart matrices and performance analysis of MIMO MRC systems," IEEE J. Sel. Areas Commun., vol. 21, no. 3, pp. 418-426, Apr. 2003.

[19] S. Jin, M. R. McKay, X. Gao, and I. B. Collings, "MIMO multichannel beamforming: SER and outage using new eigenvalue distribution of complex noncentral Wishart matrices," IEEE Trans. Commun., vol. 56, no. 3, pp. 424-434, Mar. 2008. 
[20] R. H. Y. Louie, M. R. McKay, and I. B. Collings, "Impact of correlation on the capacity of multiple access and broadcast channels with MIMOMRC," IEEE Trans. Wireless Commun., vol. 7, no. 6, pp. 2397-2407, June 2008.

[21] A. Zanella, M. Chiani, and M. Z. Win, "Performance of MIMO MRC in correlated Rayleigh fading environments," in Proc. IEEE Veh. Technol. Conf. (VTC), Stockholm, Sweden, vol. 3, May 2005, pp. 1633-1637.

[22] L. G. Ordóñez, D. P. Palomar, and J. R. Fonollosa, "Ordered eigenvalues of a general class of Hermitian random matrices with application to the performance analysis of MIMO systems," IEEE Trans. Signal Process., vol. 57, no. 2, pp. 672-689, Feb. 2009.

[23] A. Zanella, M. Chiani, and M. Z. Win, "On the marginal distribution of the eigenvalues of Wishart matrices," IEEE Trans. Commun., vol. 57, no. 4, pp. 1050-1060, Apr. 2009.

[24] A. Zanella and M. Chiani, "The pdf of the lth largest eigenvalue of central Wishart matrices and its application to the performance analysis of MIMO systems," in Proc. IEEE Global Telecommun. Conf. (GLOBECOM), New Orleans, LA, Nov. 2008.

[25] A. Maaref and S. Aïssa, "Closed-form expressions for the outage and ergodic Shannon capacity of MIMO MRC systems," IEEE Trans. Commun., vol. 53, no. 7, pp. 1092-1095, July 2005.

[26] --, "Eigenvalue distributions of Wishart-type random matrices with application to the performance analysis of MIMO MRC systems," IEEE Trans. Wireless Commun., vol. 6, no. 7, pp. 2678-2689, July 2007.

[27] A. Zanella, M. Chiani, and M. Z. Win, "MMSE reception and successive interference cancellation for MIMO systems with high spectral efficiency," IEEE Trans. Wireless Commun., vol. 4, no. 3, pp. 1244-1253, May 2005.

[28] A. M. Tulino and S. Verdú, "Random matrix theory and wireless communications," Foundations Trends Commun. Inf. Theory, vol. 1, no. 1, pp. 1-163, 2004.

[29] J. W. Demmel, "The probability that a numerical analysis problem is difficult," Math. Comput., vol. 50, pp. 449-480, 1988.

[30] V. Erceg, P. Soma, D. S. Baum, and A. J. Paulraj, "Capacity obtained from multiple-input multiple-output channel measurements in fixed wireless environments at $2.5 \mathrm{GHz}$," in Proc. IEEE Int. Conf. Commun. (ICC), vol. 1, New York, May 2002, pp. 396-400.

[31] M. D. Batariere et al., "Wideband MIMO mobile impulse response measurements at $3.7 \mathrm{GHz}$," in Proc. IEEE Veh. Technol. Conf. (VTC), Birmingham, AL, May 2002, pp. 26-30.

[32] H. Artes, D. Seethaler, and F. Hlawatsch, "Efficient detection algorithms for MIMO channels: a geometrical approach to approximate ML detection," IEEE Trans. Signal Process., vol. 51, no. 11, pp. 2808-2820, Nov. 2003.

[33] D. Wubben, R. Bohnke, V. Kuhn, and K. D. Kammeyer, "MMSE-based lattice-reduction for near-ML detection of MIMO systems," in Proc. ITG Work. Smart Antennas (WSA), Munich, Germany, Mar. 2004, pp. 106-113.

[34] J. Maurer, G. Matz, and D. Seethaler, "Low-complexity and fulldiversity MIMO detection based on condition number thresholding," in Proc. IEEE Int. Conf. Acoustics Speech Signal Process. (ICASSP), vol. 3, Honolulu, HI, Apr. 2007, pp. 61-64.

[35] Y. Zeng and Y.-C. Liang, "Eigenvalue based spectrum sensing algorithms for cognitive radio," IEEE Trans. Commun., vol. 57, no. 6, pp. 1784-1793, June 2009.

[36] F. Penna, R. Garello, and M. Spirito, "Cooperative spectrum sensing based on the limiting eigenvalue ratio distribution in Wishart matrices," IEEE Commun. Lett., vol. 13, no. 7, pp. 507-509, July 2009.

[37] L. S Cardoso, M. Debbah, P. Bianchi, and J. Najim, "Cooperative spectrum sensing using random matrix theory," in Proc. IEEE Int. Symp. Wireless Pervasive Comput. (ISWPC), Santorini, Greece, May 2008, pp. 334-338.

[38] G. Strang, Linear Algebra and its Applications, 3rd edition. San Diego: Harcourt Brace Jovanovich Inc., 1988.

[39] R. W. Heath, Jr. and A. J. Paulraj, "Switching between diversity and multiplexing in MIMO systems," IEEE Trans. Commun., vol. 53, no. 6, pp. 962-968, June 2005.

[40] T. H. Chan, M. Hambi, C. Y. Cheung, and M. Ma, "A link adaptation algorithm in MIMO-based WiMAX systems," J. Commun., vol. 2, no. 5, pp. 16-24, Aug. 2007.

[41] A. Edelman, "Eigenvalues and condition numbers of random matrices," Ph.D dissertation, MIT, Cambridge, MA, May 1989.

[42] Z. Chen and J. J. Dongarra, "Condition numbers of Gaussian random matrices," SIAM J. Matrix Anal. Applic., vol. 27, no. 3, pp. 603-620, July 2005.

[43] J.-M. Azaïs and M. Wschebor, "Upper and lower bounds for the tails of the distribution of the condition number of a Gaussian matrix," SIAM J. Matrix Anal. Applic., vol. 26, no. 2, pp. 426-440, 2005.
[44] A. Edelman and B. D. Sutton, "Tails of condition number distributions," SIAM J. Matrix Anal. Applic., vol. 27, no. 2, pp. 547-560, 2005.

[45] F. Penna, R. Garello, D. Figlioli, and M. A. Spirito, "Exact nonasymptotic threshold for eigenvalue-based spectrum sensing," in Proc. IEEE Int. Conf. Cognit. Radio Oriented Wireless Netw. Commun. (CROWNCOM), Hannover, Germany, June 2009.

[46] M. Chiani and A. Zanella, "Joint distribution of an arbitrary subset of the ordered eigenvalues of Wishart matrices," in Proc. IEEE Pers. Indoor Mobile Radio Conf. (PIMRC), Cannes, France, Sep. 2008.

[47] T. Ratnarajah, R. Vaillancourt, and M. Alvo, "Eigenvalues and condition numbers of complex random matrices," SIAM J. Matrix Anal. Applic., vol. 26, no. 2, pp. 441-456, Jan. 2005.

[48] M. Matthaiou, D. I. Laurenson, and C.-X. Wang, "On analytical derivations of the condition number distributions of dual non-central Wishart matrices," IEEE Trans. Wireless Commun., vol. 8, no. 3, pp. 1212-1217, Mar. 2009.

[49] L. Zheng and D. N. C. Tse, "Diversity and multiplexing: a fundamental tradeoff in multiple-antenna channels," IEEE Trans. Inf. Theory, vol. 49, no. 5, pp. 1073-1096, May 2003.

[50] A. T. James, "Distributions of matrix variates and latent roots derived from normal samples," Ann. Math. Stat., vol. 35, no. 2, pp. 475-501, June 1964.

[51] W. Y. Tan and R. P. Gupta, "On approximating the non-central Wishart distribution with Wishart distribution," Commun. Stat. Theory Method, vol. 12, no. 22, pp. 2589-2600, 1983.

[52] H. Gao and P. J. Smith, "A determinant representation for the distribution of quadratic forms in complex normal vectors," Elsevier J. Mult. Anal., vol. 73 , no. 2, pp. 155-165, May 2000.

[53] I. S. Gradshteyn and I. M. Ryzhik, Table of Integrals, Series, and Products, 6th edition. San Diego: Academic Press, 2000.

[54] A. H. Nuttall, "Some integrals involving the $Q$-function," Naval Underwater Systems Center, New London Lab., New London, CT, 1972.

[55] M. K. Simon, "The Nuttall $Q$ function-its relation to the Marcum $Q$ function and its application in digital communication performance evaluation," IEEE Trans. Commun., vol. 50, no. 11, pp. 1712-1715, Nov. 2002.

[56] J. Jaldén, D. Seethaler, and G. Matz, "Worst- and average-case complexity of LLL lattice reduction in MIMO wireless systems," in Proc. IEEE Int. Conf. Acoustics Speech Signal Process. (ICASSP), Las Vegas, NV, Mar. 2008, pp. 2685-2688.

[57] Z. Bai et al., Templates for the Solution of Algebraic Eigenvalue Problems: A Practical Guide. Philadelphia: SIAM, 2000.

[58] N. Kita, W. Yamada, A. Sato, D. Mori, and S. Uwano, "Measurement of Demmel condition number for $2 \times 2$ MIMO-OFDM broadband channels," in Proc. IEEE Veh. Technol. Conf. (VTC), Milan, Italy, May 2004, pp. 294-298.

[59] P.-H. Kuo, "Channel variations in MIMO wireless communication systems: eigen-structure perspectives," Ph.D. dissertation, University of Canterbury, Christchurch, New Zealand, June 2007.

[60] O. Besson and L. L. Scharf, "CFAR matched direction detector," IEEE Trans. Signal Process., vol. 54, no. 7, pp. 2840-2844, July 2006.

[61] S. L. Loyka, "Channel capacity of MIMO architecture using the exponential correlation matrix," IEEE Commun. Lett., vol. 5, no. 9, pp. 369-371, Sep. 2001.

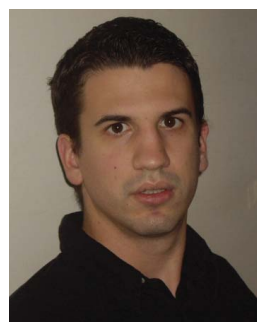

Michail Matthaiou (S'05-M'08) was born in Thessaloniki, Greece in 1981. He obtained the Diploma degree in Electrical and Computer Engineering from the Aristotle University of Thessaloniki, Greece in 2004. He then received the M.Sc. (with distinction) in Communication Systems and Signal Processing from the University of Bristol, U.K. and Ph.D. degrees from the University of Edinburgh, U.K. in 2005 and 2008, respectively. Since September 2008, he has been with the Institute for Circuit Theory and Signal Processing, Munich University of Technology (TUM), Germany working as a Postdoctoral Research Associate. During the summer of 2009 , he was a visiting researcher at University of WisconsinMadison, WI. His research interests span signal processing for wireless communications, random matrix theory, multi-dimensional array processing and MIMO channel modeling.

Dr. Matthaiou is a co-recipient of the 2006 IEEE Communications Chapter Project Prize for the best M.Sc. dissertation in the area of communications. 


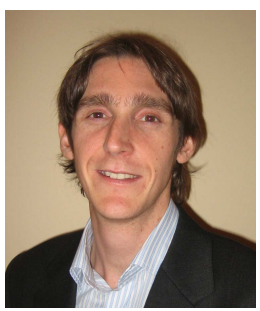

Matthew R. McKay (S'03-M'07) received the combined B.E. degree in electrical engineering and B.IT. degree in computer science from the Queensland University of Technology, Australia, in 2002, and the Ph.D. degree in electrical engineering from the University of Sydney, Australia, in 2006.

He then worked as a Research Scientist at the Commonwealth Science and Industrial Research Organization (CSIRO), Sydney, prior to joining the faculty at the Hong Kong University of Science and Technology (HKUST) in 2007, where he is currently an Assistant Professor. He is also a member of the Center for Wireless Information Technology at HKUST. His research interests include communications and signal processing; in particular, the analysis and design of MIMO systems, random matrix theory, information theory, and wireless ad hoc and sensor networks.

Dr. McKay was awarded a 2006 Best Student Paper Award at IEEE ICASSP'06, and was jointly awarded the 2006 Best Student Paper Award at IEEE VTC'06-Spring. He was also awarded the University Medal upon graduating from the Queensland University of Technology.

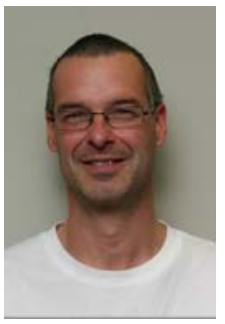

Peter J. Smith received the B.Sc degree in Mathematics and the Ph.D degree in Statistics from the University of London, London, U.K., in 1983 and 1988, respectively. From 1983 to 1986 he was with the Telecommunications Laboratories at GEC Hirst Research Centre. From 1988 to 2001 he was a lecturer in statistics at Victoria University, Wellington, New Zealand. Since 2001 he has been a Senior Lecturer and Associate Professor in Electrical and Computer Engineering at the University of Canterbury in New Zealand. His research interests include the statistical aspects of design, modeling and analysis for communication systems, especially antenna arrays, MIMO, cognitive radio and relays.

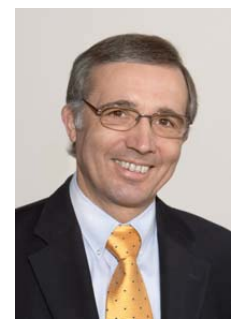

Josef A. Nossek (S'72-M'74-SM'81-F'93) received the Dipl.-Ing. and the Dr. techn. degrees in electrical engineering from the University of Technology in Vienna, Austria in 1974 and 1980, respectively. In 1974 he joined Siemens AG in Munich, Germany as a member of technical staff, in 1978 he became supervisor, and from 1980 on he was Head of Department. In 1987 he was promoted to be Head of all radio systems design. Since 1989 he has been Full Professor for circuit theory and signal processing at the Munich University of Technology where he teaches undergraduate and graduate courses on circuit and systems theory and signal processing and leads research on signal processing algorithms for communications. He was President Elect, President and Past President of the IEEE Circuits and Systems Society in 2001, 2002 and 2003 respectively. He was Vicepresident of VDE (Verband der Elektrotechnik, Elektronik und Informationstechnik e.V.) 2005 and 2006, President of VDE 2007 and 2008 and is again Vicepresident of VDE in 2009 and 2010. His awards include the ITG Best Paper Award 1988, the Mannesmann Mobilfunk (now Vodafone) Innovationsaward 1998, the Award for Excellence in Teaching from the Bavarian Ministry for Science, Research and Art in 1998 and the Golden Jubilee Medal of the IEEE Circuits and Systems Society for 'Outstanding Contributions to the Society' in 1999. In 2008 he received the Education Award of the IEEE Circuits and Systems Society and the Order of Merit from the the Federal Republic of Germany. Since 2009 he is Member of the National Academy of Engineering Sciences of Germany (acatech). 